\title{
PolSAR Time Series Processing With Binary Partition Trees
}

\author{
Alberto Alonso-González, Student Member, IEEE, Carlos López-Martínez, Senior Member, IEEE, and \\ Philippe Salembier, Fellow, IEEE
}

\begin{abstract}
This paper deals with the processing of polarimetric synthetic aperture radar (SAR) time series. Different approaches to deal with the temporal dimension of the data are considered, which are derived from different target characterizations in this dimension. These approaches are the basis for defining two different binary partition tree (BPT) structures that are employed for SAR polarimetry (PolSAR) data processing. Once constructed, the BPT is processed by a tree pruning, producing a set of spatiotemporal homogeneous regions, and estimating the polarimetric response within them. It is demonstrated that the proposed technique preserves the PolSAR information in the spatial and the temporal domains without introducing bias nor distortion. Additionally, the evolution of the data in the temporal dimension is also analyzed, and techniques to obtain BPT-based scene change maps are defined. Finally, the proposed techniques are employed to process two real RADARSAT-2 data sets.
\end{abstract}

Index Terms-Binary partition tree (BPT), change detection, synthetic aperture radar (SAR) polarimetry, segmentation, time series.

\section{INTRODUCTION}

D URING recent years, radar remote sensing has improved significantly in terms of quality and quantity. Synthetic aperture radar (SAR) is a technique to achieve high spatial resolution by coherently processing different radar echoes received by a moving sensor at different positions. SAR systems may generate images of the observed area complex reflectivity independently of the weather and the day-night cycle, and additionally, when they are mounted on board a space platform, they can achieve global coverage. SAR polarimetry (PolSAR) is a type of multidimensional SAR data, where different wave polarizations are considered for the transmitted and received echoes. PolSAR has demonstrated during the last decade its usefulness to study and characterize the Earth surface, due to its ability to retrieve biophysical and geophysical information [1]-[4].

Manuscript received November 15, 2012; revised April 15, 2013; accepted July 7, 2013. This work was supported in part by the MICINN TEC Project MUSEO (TEC2011-28201-C02-01) and the CUR of the DIUE of the Autonomous Government of Catalonia and the European Social Fund. Flevoland data were provided by the European Space Agency in the frame of the AgriSAR 2009 campaign. Barcelona data were provided by MDA through the Project SOAR-EU 6779.

The authors are with the Department of Signal Theory and Communications (TSC), Technical University of Catalonia (UPC), 08034 Barcelona, Spain (e-mail: alberto.alons@gmail.com; carlos.lopez@tsc.upc.edu; philippe. salembier@upc.edu).

Color versions of one or more of the figures in this paper are available online at http://ieeexplore.ieee.org.

Digital Object Identifier 10.1109/TGRS.2013.2273664
Typically, in SAR systems, the spatial resolution is larger than the wavelength, resulting in a measure that is the coherent sum of all the individual targets present within each resolution cell. In the case of distributed scatterers, this combination may be constructive and destructive, and although it is an electromagnetic measure determined by the individual target distribution, it is considered as speckle noise, as it can only be characterized statistically. The speckle is an important drawback when processing SAR data, making a speckle filtering process necessary in order to obtain a reliable characterization of the scene reflectivity. This fact leads to some difficulties for the classical pixel-based image interpretation. Each pixel represents a very localized area and is, in general, contaminated by speckle noise. Consequently, it usually contains a small amount of valuable information. In order to extract useful information from the scene, several pixels have to be combined. A linear filtering may be applied to the data in order to reduce the effect of the speckle, but to obtain useful information, only homogeneous pixels should be combined, i.e., pixels corresponding to the same physical target distribution. In recent years, some filtering techniques have been developed, trying to avoid mixing inhomogeneous samples by adapting the filter region of support to the spatial contours of the image. These adaptation can be performed by some predefined directional windows [5]. More recent methods employ a region growing technique to obtain an arbitrary homogeneous neighborhood for each image pixel [6]. To address this issue, in this paper, instead of working directly with the original image pixels, we propose to employ a region-based and multiscale data abstraction, the binary partition tree (BPT) [7].

Nowadays, the growing presence of spaceborne SAR systems has empowered the collection of temporal series data sets, containing different acquisitions of the same scene collected at distinct time instants. These data sets contain useful information related not only to the scene but also to its temporal evolution and dynamics. However, the analysis and exploitation of this information is a big challenge that is now starting to be studied and developed. Some recent contributions have tried to face this problem in several ways. In the field of optical imagery, some techniques have tackled with these data sets [8], [9]. For single-polarization SAR, in [10], temporal series are employed to filter, in spatial and temporal dimensions, pixels of different acquisitions and obtain a better quality image. This information has also been employed for terrain classification [11], taking into account the temporal variability and the long-term coherence. On a more recent contribution, in [12], PolSAR satellite image time series mining is proposed based 
on the temporal evolution of some target polarimetric parameter information.

In this paper, the processing and analysis of PolSAR time series is addressed, based on a common BPT framework allowing different approaches for data modeling. These approaches differ basically on the assumption of the target characterization in the temporal dimension, and this will lead also to different modus operandi for data processing and analysis. Depending on the target characterization, two different BPT data representations will be derived, and the strengths and limitations of both approaches will be studied in detail. Moreover, a novel temporal stability measure based on distances is proposed, exploiting the complete polarimetric information under the Gaussian hypothesis, which is employed for change detection over real data and compared with ground-truth information.

This paper is structured as follows. Section II introduces the BPT structure that will be used to process the data. In Section III, the different approaches to model the temporal dimension of the data will be presented, including the different region models needed to characterize the regions of the scene. Section IV presents the details of the BPT construction approaches that will be employed to process PolSAR time series under the different temporal assumptions. The processing and analysis of BPTs is described in Section V, whereas the results over real data are presented in Section VI. In Section VII, the temporal information of the results is analyzed to extract information related to the scene evolution and change detection, comparing results with ground-truth information and contrasting them with other state-of-the-art PolSAR change detection techniques. Finally, Section VIII closes this paper discussing the obtained results and indicating other potentialities of the proposed techniques.

\section{BPT REPRESENTATION}

Instead of working directly with the original image pixels, we propose to employ a BPT [7] as a PolSAR data abstraction for data processing and information extraction. The BPT is a region-based multiscale data representation. Each node of the tree represents a connected region of the data. The leaf nodes of the tree represent single pixels of the original data, whereas the remaining nodes represent the regions produced by the merging of the neighboring regions represented by its two child nodes. As a consequence, the root node of the BPT represents the whole data set. Note that, between the leaves and the root node, there is a wide number of regions representing areas of the original data set having similar values at different scales.

When processing SAR data, as mentioned before, the effect of the speckle noise makes some filtering necessary. A linear filter may be applied to the data, but then, the obtained results are only valid over locally stationary areas and SAR data are strongly inhomogeneous, as they reflect the complexity of the scene. The BPT may be useful in this scenario, by identifying homogeneous regions of the data and applying this filtering only within them. It is assumed then that, although SAR data are inhomogeneous, they may be divided into homogeneous regions. Additionally, due to the multiscale nature of the BPT, regions of completely different sizes may be obtained, pre-
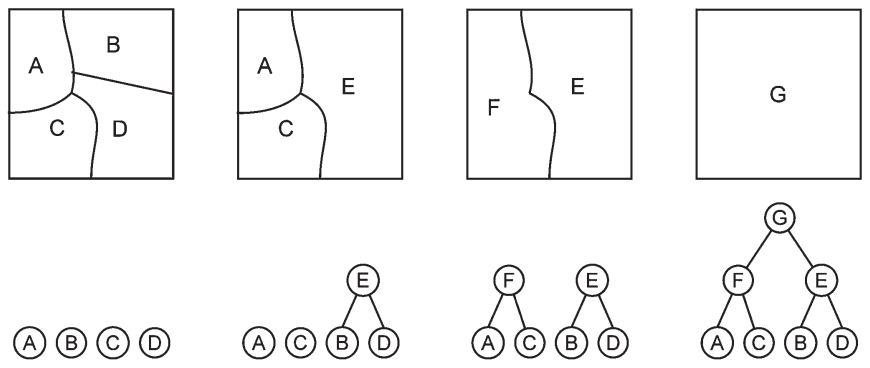

Fig. 1. BPT construction example with four initial regions.

serving the local information of isolated targets while also maintaining the global behavior of large areas. The ability of the BPT to describe the data at multiple scales makes it suitable to support applications such as classification, segmentation, or information retrieval.

In order to represent a region of the data, a region model is associated to each node to summarize useful and relevant information about the region that it is describing. Note that the BPT structure is not tied to any particular model. However, in order to generate a valuable data abstraction, the employed region model should be representative enough to describe the data for all the applications for which the BPT is intended to be used.

As proposed in [7], the BPT representation may be constructed by an iterative merging algorithm in a bottom-up approach. Starting from the leaves of the tree, at every construction step, the two most similar neighboring regions are merged until the root of the tree is generated. An example of the BPT construction process may be seen in Fig. 1 from a data set with four initial regions.

In order to apply this construction process, the following elements have to be defined.

1) Data connectivity. Assuming that the original data set is composed of pixels, a connectivity has to be defined over them, on top of which the connected regions will be generated.

2) Region model. As stated before, each region should be represented by a model containing the most relevant region information.

3) Similarity measure. The BPT construction process is based on merging the two most similar neighboring regions at each construction step. Therefore, a measure has to be defined over the region model space in order to quantify the similarity between regions.

The similarity measure should ideally take into account all the knowledge about the region model space. In fact, this is the keystone of the construction process since it defines the merging sequence and the final BPT structure. The regions of the tree represent areas of the image having similar model values, according to that measure, at different scales. Note that the BPT representation only exploits the internal relationships within the data, and as a result, it is application independent.

This construction algorithm performs a number of iterations proportional to the initial nodes. Note that a binary tree having $n$ leaves contains $2 n-1$ nodes [13]. Consequently, this algorithm has to perform $n-1$ fusions to fully generate the BPT. However, at each iteration, it has to look for the pair of 


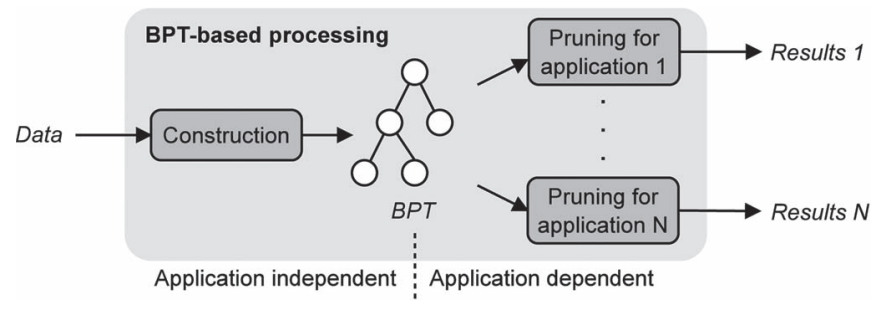

Fig. 2. BPT-based processing scheme.

nodes with the higher similarity and recompute the similarities involving the generated node. Assuming that the number of neighbors per region may be bounded by a constant $k_{n}$, thus not depending on the data set size, and employing some efficient data structures for storing and sorting region similarities, the computational complexity of the BPT construction algorithm may be bounded to $O\left(k_{n} n \cdot \log n\right)=O(n \cdot \log n)$, where $n$ is the number of pixels of the original data set.

Once the BPT representation has been constructed, it has to be exploited for a particular application. This task may be performed by a tree cut or pruning process [7]. The pruning process may be seen as the extraction of the useful or interesting regions from the tree for a particular application. Consequently, the pruning process is application dependent. The whole BPTbased processing chain may be seen in Fig. 2. Note that the BPT construction process, as it is application independent, only has to be performed once.

The pruning algorithm has to traverse, at most, the $2 n-1$ nodes of the BPT. If the evaluation cost of the pruning criterion is constant $k_{p}$, the computational complexity of the pruning algorithm is $O\left(k_{p}(2 n-1)\right)=O(n)$.

\section{PolSAR Time Series Processing Approaches}

In order to include the temporal information, two different approaches are considered. To simplify the processing, it is assumed that all the acquisitions are coregistered. The two approaches differ in the way that the full data set, containing all the acquisitions, is divided into its individual data elements to be processed.

On the one hand, it can be assumed that a target may be characterized by the statistical behavior of its polarimetric response $\mathrm{k}$ measured by the radar

$$
\mathbf{k}=\left[\begin{array}{lll}
S_{h h} & \frac{\sqrt{2}}{2}\left(S_{h v}+S_{v h}\right) & S_{v v}
\end{array}\right]^{T}
$$

where $S_{i j}$ represents the target scattering coefficient for $i$, $j \in[h, v]$ transmitted and received polarization combination, assuming a monostatic radar, $h$ and $v$ stand for the horizontal and vertical polarizations, respectively, and ${ }^{T}$ stands for the vector transpose.

Accordingly, the target response is characterized by its sample covariance matrix $\mathbf{Z}$, which contains all the needed information to characterize the target, under the Gaussian hypothesis

$$
\mathbf{Z}=\left\langle\mathbf{k k}^{H}\right\rangle_{n}=\frac{1}{n} \sum_{i=1}^{n} \mathbf{k}_{i} \mathbf{k}_{i}^{H}
$$

where $\mathbf{k}_{i}$ represents the scattering vector of the $i$ th pixel, $n$ represents the number of pixels averaged, and ${ }^{H}$ represents the complex Hermitian transpose.

Hence, a change in the polarimetric response is assumed to correspond to a target change. Under this approach, the individual data elements are the pixels of every acquisition. Consequently, the addition of the temporal dimension to the data set does not change the data element considered, and then, the time dimension is treated as an additional dimension of the data. In this case, the resulting data set containing $N$ acquisitions having $n_{r}$ and $n_{a}$ pixels each in range and azimuth, respectively, is composed of $n_{r} \times n_{a} \times N$ elements. Then, a 3-D data set has to be processed under this approach.

On the other hand, it can be assumed that a target is characterized by the temporal evolution of its polarimetric response among all the acquisitions. Then, the different acquisitions are different realizations of the same target response at different time instants, which may be characterized by the extended scattering vector $\mathbf{k}_{e}$

$$
\mathbf{k}_{e}=\left[\begin{array}{llll}
\mathbf{k}_{1}^{T} & \mathbf{k}_{2}^{T} & \ldots & \mathbf{k}_{N}^{T}
\end{array}\right]^{T}
$$

where $\mathbf{k}_{i}$ represents the scattering vector at the $i$ th acquisition, as defined in (1), and $N$ represents the number of acquisitions in the data set.

Then, one may characterize each target by its response evolution statistics among the temporal dimension

$$
\mathbf{Z}_{e}=\left\langle\mathbf{k}_{e} \mathbf{k}_{e}^{H}\right\rangle_{n}=\left(\begin{array}{cccc}
\mathbf{Z}_{11} & \boldsymbol{\Omega}_{12} & \cdots & \boldsymbol{\Omega}_{1 N} \\
\boldsymbol{\Omega}_{12}^{H} & \mathbf{Z}_{22} & \cdots & \boldsymbol{\Omega}_{2 N} \\
\vdots & \vdots & \ddots & \vdots \\
\boldsymbol{\Omega}_{1 N}^{H} & \boldsymbol{\Omega}_{2 N}^{H} & \cdots & \mathbf{Z}_{N N}
\end{array}\right)
$$

where $N$ represents the number of acquisitions of the data set, $\mathbf{Z}_{i i}$ is a 3 by 3 covariance matrix representing the polarimetric information of the $i$ th acquisition, as expressed in (2), and $\boldsymbol{\Omega}_{i j}$ is a 3 by 3 complex matrix representing the correlation between the acquisitions $i$ and $j$.

Under this approach, the individual data elements are not individual pixels, as in the previous approach, but the set of collocated pixels for all acquisition time instants. These individual data elements are described by (4). Consequently, the addition of the temporal dimension to the data set enlarges the considered data element model. In other words, the time dimension is employed to improve the characterization of each scene location. In this case, the resulting data set is composed of $n_{r} \times n_{a}$ individual elements. Then, unlike the previous case, a 2-D data set is processed under this approach.

\section{PolSAR TIME SERIES BPT}

The BPT representation can be employed to process PolSAR images, as described in [14]-[16]. In these publications, the traditional 8-connectivity was employed, and the polarimetric covariance matrix (2) was used as a region model.

The employment of the estimated covariance matrix $\mathbf{Z}$ is convenient for the BPT representation since most of the PolSAR applications and parameter inversion models are based on this statistical descriptor. Moreover, assuming the complex 
Gaussian model for PolSAR data, the region model in (2) contains all the information needed to completely define the statistical behavior of the regions. However, note that this is only true assuming that there is no region mixture. In the tree nodes that are close to the root and that cover large areas of the scene, this hypothesis may not be true, resulting in a region model that is not capable to describe properly these areas. Nonetheless, for the applications of interest of this paper, the BPT representation relying on the proposed region models is still useful if we are only interested in homogeneous regions of the data.

To apply the described BPT construction process, a similarity measure over the region model space has to be defined. Several measures over the estimated covariance matrices $\mathbf{Z}$ have been proposed and analyzed in [15] and [17], and $d_{s g}$, adapted to the positive definite matrix cone geometry [18], led to the best performances, producing larger homogeneous regions and minimizing oversegmentation

$$
d_{s g}(X, Y)=\left\|\log \left(\mathbf{Z}_{X}^{-1 / 2} \mathbf{Z}_{Y} \mathbf{Z}_{X}^{-1 / 2}\right)\right\|_{F}+\ln \left(\frac{2 n_{x} n_{y}}{n_{x}+n_{y}}\right)
$$

where $\mathbf{Z}_{X}$ and $\mathbf{Z}_{Y}$ represent the estimated covariance matrices for regions $X$ and $Y$, respectively, $n_{x}$ and $n_{y}$ represent their number of pixels, $\|\cdot\|_{F}$ represents the Frobenius matrix norm, $\log ($.$) represents the matrix \operatorname{logarithm}$, and $\ln ($.$) represents the$ natural logarithm.

In this paper, this BPT representation initially developed for single images is adapted to process PolSAR temporal series data sets, having different acquisitions of the same scene at different dates. Therefore, the temporal dimension is added to the data set. On these data sets, we are interested not only in the polarimetric information of the scene but also in its temporal evolution among all the different acquisitions. Two different alternatives to deal with this temporal dimension are proposed, based on different assumptions of the data behavior among this dimension, as presented in Section III, that lead to two different BPT representations.

\section{A. ST BPT}

The BPT may be extended to the temporal dimension by assuming that it is an additional independent dimension of the data [19], as presented in the first approach of Section III. In this approach, the same region model as for a PolSAR image is employed, which is the 3 by 3 estimated covariance matrix (2), which describes its polarimetric response. Consequently, 3 -D regions are obtained, representing pixels of the data set having similar polarimetric response. According to Section II, the following elements are defined.

1) Data connectivity. The 10-connectivity [19], as shown in Fig. 3, is employed. Each pixel is connected to the eight neighboring pixels in the same acquisition and the pixels on the same position in the acquisitions just before and after.

2) Region model. As previously stated, each region is modeled by the sample covariance matrix $\mathbf{Z}$.

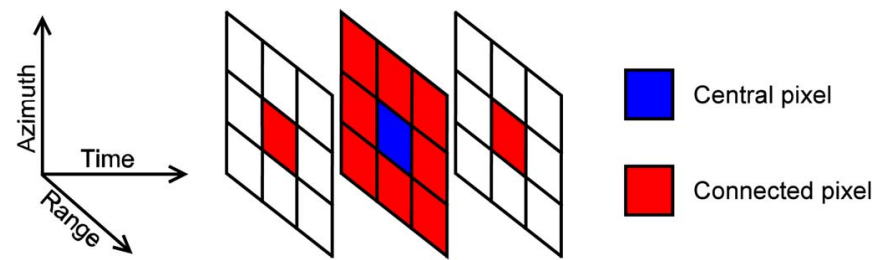

Fig. 3. ST BPT pixel connectivity.

3) Similarity measure. Since the region model is the same as in the single PolSAR image BPT representation, the same similarity measures may be employed, as the $d_{s g}$ measure defined in (5).

The resulting data abstraction represents connected 3-D space-time regions of the data having similar polarimetric response. Hence, in this approach, the temporal evolution of this information may be found on the region contours in the temporal dimension. A region contour between different acquisitions in the same location may be interpreted as a polarimetric response change, assumed as a target change. On the contrary, if there is no change, these pixels in the same location at different acquisitions belong to the same region, and they are represented by the same region model. Note that this approach then is averaging similar samples of different acquisitions when computing the region model, as indicated in (2), preventing the exploitation of the interferometric information between acquisitions. On the other hand, it has the advantage of increasing the amount of averaged samples, thus reducing the effect of the speckle noise and improving the polarimetric information estimation.

\section{B. TE BPT}

Another possible modus operandi to deal with the temporal dimension is to include it within the region model [20], [21], as presented in the second approach of Section III. Then, instead of modeling each region by its polarimetric response (2), an extended model is defined to include also its temporal evolution. In this paper, the extended estimated covariance matrix $\mathbf{Z}_{e}(4)$ is employed.

In this approach, since the temporal evolution is included within the region model, the BPT represents spatial regions having a similar polarimetric temporal evolution among all the acquisitions. In the following, this representation will be referred to as temporal evolution BPT (TE BPT), which corresponds to the structure called extended BPT in [20] and [21]. As opposite to the space-time BPT (ST BPT), where the temporal evolution may be found in the region contours on this dimension, in this representation, this evolution is stored directly within the region model, in the $\mathbf{Z}_{i i}$ matrices.

As mentioned in Section II, the following elements have to be defined for the BPT construction process.

1) Data connectivity. In the TE BPT, the temporal information has been included within the region model, and the resulting data to be merged are therefore 2-D. Consequently, the classical 8-connectivity [15] may be applied.

2) Region model. As mentioned before, the extended estimated covariance matrix $\mathbf{Z}_{e}$ (4) is employed. 
3) Similarity measure. As explained before, the $d_{s g}$ measure provides the best results for single PolSAR image BPT. Accordingly, a modification of (5) is proposed to employ it for the extended covariance matrix $\mathbf{Z}_{e}$ region model defined in (4)

$$
d_{g}(X, Y)=\sqrt{\sum_{i=1}^{N}\left\|\log \left(\mathbf{Z}_{X_{i i}}^{-1 / 2} \mathbf{Z}_{Y_{i i}} \mathbf{Z}_{X_{i i}}^{-1 / 2}\right)\right\|_{F}^{2}}+\ln \left(\frac{2 n_{x} n_{y}}{n_{x}+n_{y}}\right)
$$

where $\mathbf{Z}_{X_{i i}}$ represents the $\mathbf{Z}_{i i}$ component of the $X$ region, as shown in (4). The same idea may also be applied to extend other similarity measures.

Note that the $d_{g}$ measure defined in (6) employs only the polarimetric information contained within the $\mathbf{Z}_{i i}$ matrices, ignoring the information contained within $\boldsymbol{\Omega}_{i j}$. In fact, the $d_{s g}$ measure (5) could be applied directly to the full $\mathbf{Z}_{e}$ matrix, but this will have some drawbacks and limitations. On the one hand, the interferometric information contained in $\Omega_{i j}$ matrices has a completely different nature and interpretation than $\mathbf{Z}_{i i}$, depending on spatial baselines, topography, subsidence, etc. [22]. On the other hand, the $d_{s g}$ measure requires a full-rank $\mathbf{Z}$ matrix to be computed, which requires for the extended model $\mathbf{Z}_{e}$ an initial filtering or regularization process with at least $3 N$ independent samples, which may become a high number even for a small number of acquisitions $N$. Alternatively, the $d_{g}$ measure employs the full polarimetric temporal evolution information and only requires a regularization with at least three independent samples.

It is worth noticing that, for a single acquisition, having $N=1$, the two approaches defined in Sections IV-A and IV-B are exactly the same, corresponding also to the PolSAR BPT representation presented in [15].

Concerning the computational complexity of both approaches, the number of nodes on the resulting BPT is different in both cases. The ST BPT contains $N n$ leaves, whereas the TE BPT contains $n$ leaves, where $n$ is the number of pixels in one of the $N$ acquisitions of the data set. Nevertheless, although the TE BPT contains $n$ leaf nodes, the cost of computing the similarity measures increases linearly with $N$, as shown in (6). Consequently, the computational complexity for both cases will be $O(N n \cdot \log N n)$ for the ST BPT and $O(N n \cdot \log n)$ for the TE BPT.

\section{PolSAR Time SERIES BPT PRUNing}

To extract useful information from the BPT representation, a tree pruning process is employed, as mentioned in Section II. For the speckle filtering application and to obtain a good target response estimation, the goal is to extract the biggest homogeneous regions from the tree. Consequently, a region homogeneity-based pruning may be employed, similarly to the single PolSAR acquisition case in [15]. This pruning strategy is based on a region homogeneity measure $\phi$. This measure may be seen as the average error produced when representing all the data elements of a region by its region model.

For the ST BPT, a measure $\phi(X)$ based on the relative mean squared error for region $X$ is proposed [19]. The pruning process is performed by a top-down approach, starting from the root node and traversing the tree to the leaves, and the first regions having an error below a pruning threshold $\delta_{p}$ are selected

$$
\phi(X)=\frac{1}{n_{x}} \sum_{i=1}^{n_{x}} \frac{\left\|\mathbf{Z}^{i}-\mathbf{Z}_{X}\right\|_{F}^{2}}{\left\|\mathbf{Z}_{X}\right\|_{F}^{2}}<\delta_{p}
$$

where $\mathbf{Z}^{i}$ is the estimated covariance matrix for the $i$ th pixel within region $X$, having $n_{x}$ pixels, and $\delta_{p}$ is the pruning factor, usually expressed in decibels.

Note that the $\phi$ measure defined in (7) is exactly the same as for the single PolSAR acquisition BPT [15] since, in the ST BPT, there is no change on the underlying region model. In this sense, the BPT-based pruning may be considered as an abstraction of the application rationale since it is defined over the BPT representation instead of being directly defined over the data set. As shown in Fig. 2, the BPT is a middle layer in the processing scheme, separating the application-independent and application-dependent parts. Consequently, a change on the data set dimensionality, as for the ST BPT, has only impact on the BPT construction part, which deals with the original data, but it has no impact on the pruning process, which is the application-dependent part. However, note that, in this case, the regions within this BPT represent 3-D regions, including pixels of different acquisitions.

In the case of the TE BPT, presented in Section IV-B, a different pruning criterion is defined. Indeed, the $\phi(X)$ measure defined in (7) could be applied also to the $\mathbf{Z}_{e}$ matrix, but as explained in Section IV-B, it will have some limitations in capturing the temporal evolution of the data, and similarly to the $d_{g}$ similarity measure (6), a new homogeneity measure $\phi_{e}(X)$ is proposed employing only the polarimetric information

$$
\phi_{e}(X)=\frac{1}{n_{x}} \sum_{i=1}^{n_{x}} \frac{\sum_{j=1}^{N}\left\|\mathbf{Z}_{j j}^{i}-\mathbf{Z}_{X_{j j}}\right\|_{F}^{2}}{\sum_{j=1}^{N}\left\|\mathbf{Z}_{X_{j j}}\right\|_{F}^{2}}<\delta_{p}
$$

where $\mathbf{Z}_{j j}^{i}$ is the $\mathbf{Z}_{j j}$ covariance matrix for the $i$ th pixel within region $X$ and $\mathbf{Z}_{X_{j j}}$ is the $\mathbf{Z}_{j j}$ covariance matrix of the region model $\mathbf{Z}_{e}$ for the $X$ region.

The $\phi_{e}$ measure is the extension of the $\phi$ measure to the extended model (4) taking into account only the polarimetric information contained within all the $\mathbf{Z}_{i i}$ matrices. Note that it is sensitive to the full polarimetric temporal evolution information.

As mentioned in Section II, the complexity of the pruning process depends on the number of nodes of the BPT. However, note that the cost of the pruning criterion $\phi_{e}$ (8) increases linearly with $N$. Consequently, the complexity of the pruning algorithm is $O(N n)$ for both BPT approaches. It is assumed then that the pruning criterion may be computed in constant time. In fact, all the homogeneity measures $\phi$ and $\phi_{e}$ may be computed in linear time for the entire BPT. Efficiently employing the hierarchical structure, the homogeneity of each region may be calculated in constant time in terms of the homogeneity of its soon nodes. 


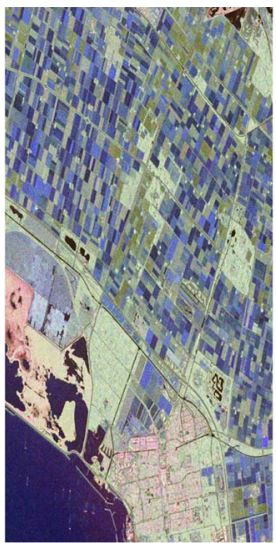

(a)

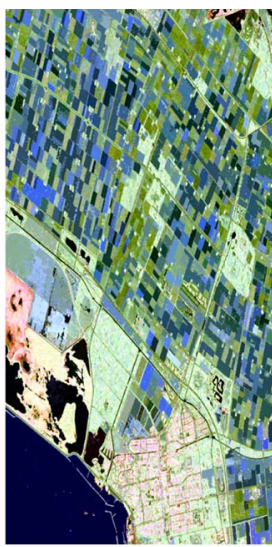

(b)

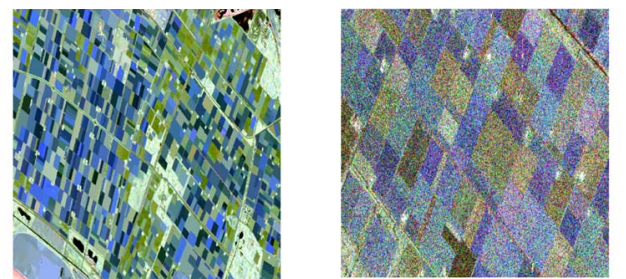

(a)

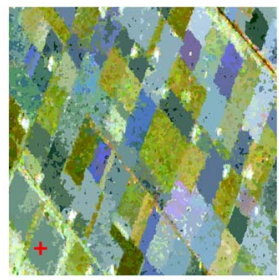

(b)

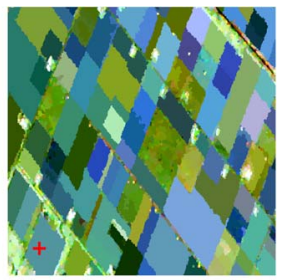

(c)
Fig. 5. Pauli representation of a crop of the second acquisition. (a) Original and after processing the full data set (b) with the ST BPT and (c) with the TE BPT. Results are shown for $\delta_{p}=-3 \mathrm{~dB}$.

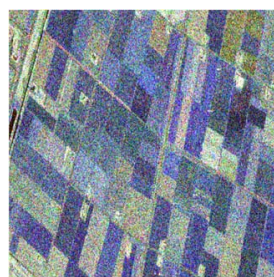

(a)

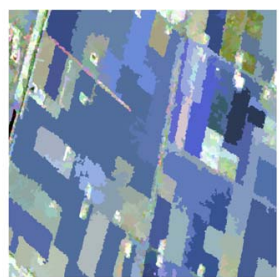

(b)

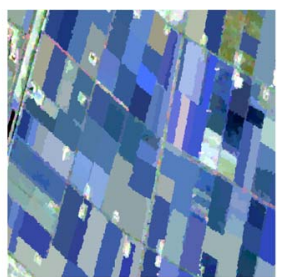

(c)
In this paper, the different proposed BPT approaches have been employed to process a real PolSAR data set. This data set contains eight RADARSAT-2 Fine Quad-Pol images corresponding to a test site in Flevoland, The Netherlands. It was acquired within the ESA AgriSAR 2009 campaign, devoted to analyze the agricultural field temporal evolution employing PolSAR data. The selected images correspond to acquisitions with the same incidence angle, employing the beam FQ13, and in ascending passes of the satellite, from April 4, 2009 to September 29, 2009, with an acquisition every 24 days. From these data, a crop having 4000 by 2000 pixels has been selected and coregistered, conforming the full Flevoland data set.

Fig. 4 shows the first acquisition of this data set and a fraction of the filtered results employing the two mentioned BPT approaches with a pruning threshold of $\delta_{p}=-3 \mathrm{~dB}$. Note that the full data set is difficult to represent for visual inspection, and then, on Fig. 4, only a portion of it, corresponding to the first acquisition, is shown, although the full data set with the eight different acquisitions has been processed. Similarly, on the following, only results over one acquisition are shown, for simplicity.

Fig. 4(b) shows the results over the first acquisition employing the ST BPT approach, whereas Fig. 4(c) shows the same results when employing the TE BPT. As it may be seen, qualitatively similar results are obtained. Both methods achieve a good speckle reduction while preserving the region contours. However, when the results are observed closely, some differences appear, particularly on the agricultural field region contours detected by both methods. Fig. 5 shows a zoom over a small $512 \times 512$ pixel area of the data set and of the previous results. In this case, the crop over the second acquisition is shown for all the cases.

As stated in [15], the proposed homogeneity-based pruning can benefit from the multiscale nature of the BPT, obtaining simultaneously large regions, corresponding to big homogeneous areas of the scene, while also maintaining the spatial resolution and preserving small details. The field contours detected when employing the TE BPT, shown in Fig. 5(c), seem
Fig. 6. Pauli representation for a detailed area of the first acquisition. (a) Original and filtered with the TE BPT employing (b) one image and (c) the full data set with $\delta_{p}=-3 \mathrm{~dB}$.

to be more precise than the ones detected with the ST BPT, shown in Fig. 5(b), when comparing them with the same area on the original image in Fig. 5(a). In fact, there is a region differentiation enhancement in this case when employing the extended model (4) with respect to the classical covariance matrix (2). This differentiation enhancement may be produced by a combination of two different reasons. On the one hand, the extended model has different realizations of the field region contours since they appear over all the different acquisitions, resulting in a noise reduction over them and making their identification easier over the extended model with respect to the simpler classical one. On the other hand, there is a differentiation enhancement between adjacent regions if they are following a different temporal evolution since this evolution is represented by the extended model, and consequently, the difference between regions gets increased if they are following this differentiation enhancement, the assumption that the region contours are spatially aligned among all the acquisitions, as the agricultural field contours, is essential. To see clearly this region differentiation enhancement, Fig. 6 shows the region contours obtained over an agricultural area on the first acquisition with the TE BPT when employing only one acquisition, having $N=1$, and when employing the full data set with $N=8$. It can be clearly seen that increasing the number of acquisitions of the scene improves the spatially fixed region contour detection.

On Figs. 4 and 5, results obtained with the ST BPT have been compared with the results obtained employing the TE BPT. However, note that these results are not fully comparable since, as shown in Sections IV-A and B, they represent different information of the scene. The TE BPT represents spatial evolution. On the contrary, the ST BPT represents regions of the data set in the space-time domain, and consequently, they have contours in both the spatial and temporal domains. a different temporal evolution. However, in order to achieve regions of the scene following a similar polarimetric temporal 


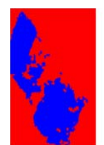

(a)

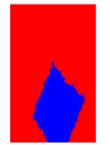

(i)

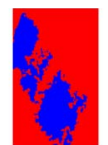

(b)

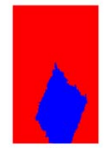

(j)

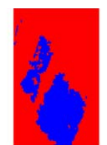

(c)

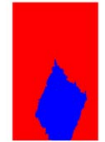

(k)

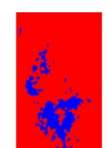

(d)

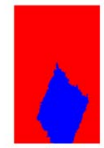

(1)

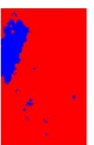

(e)

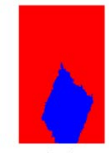

(m)

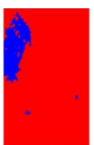

(f)

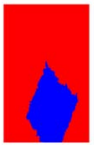

(n)

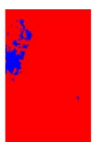

(g)

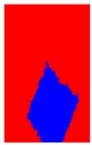

(o)

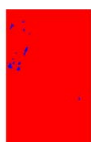

(h)

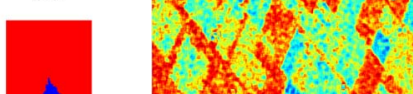

(a)

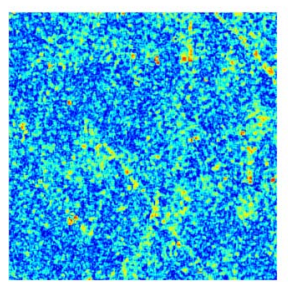

(d)

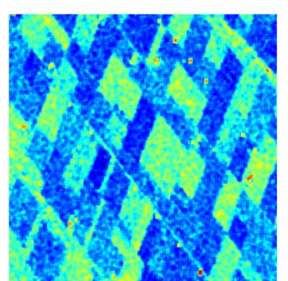

(g)

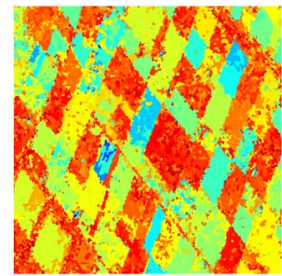

(b)

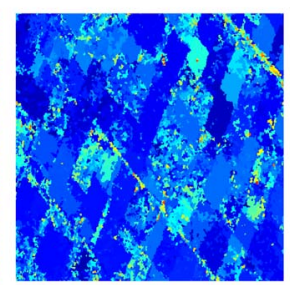

(e)

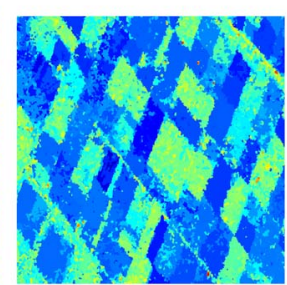

(h)

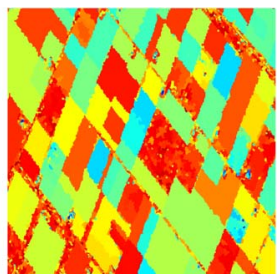

(c)

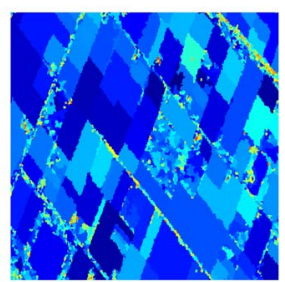

(f)

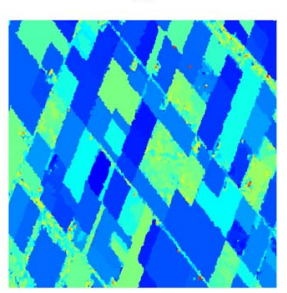

(i)

Fig. 8. H/A $/ \bar{\alpha}$ parameters of a detailed area of the second acquisition processed with the ST BPT and with the TE BPT for $\delta_{p}=-3 \mathrm{~dB}$. (a) $\mathrm{H}$, $7 \times 7$ ML. (b) H, ST BPT. (c) H, TE BPT. (d) A, $7 \times 7$ ML. (e) A, ST BPT. (f) A, TE BPT. (g) $\bar{\alpha}, 7 \times 7$ ML. (h) $\bar{\alpha}$, ST BPT. (i) $\bar{\alpha}$, TE BPT.

may substantially improve the polarimetric response estimation by combining more homogeneous samples of different acquisitions.

As a consequence, from the results shown in Figs. 4-7 and Table I, the ST BPT has the advantage to improve the number of homogeneous samples by combining different acquisitions and also the ability to adapt to scene contours that change over time, whereas the TE BPT may achieve a better detection of the spatial contours when they are fixed over time. Another advantage of the proposed extended model $\mathbf{Z}_{e}$ presented in (4) is that it can be employed to exploit the information between different acquisitions, present within the $\boldsymbol{\Omega}_{i j}$ matrices, providing a very useful Polarimetric and Interferometric SAR (PolInSAR) processing tool. Note that this information cannot be exploited from the ST BPT since it is joining information from different acquisitions.

In order to analyze the capabilities of the proposed BPT approaches to correctly estimate and preserve the polarimetric response and its evolution in the scene, the $\mathrm{H} / \mathrm{A} / \bar{\alpha}$ eigendecomposition parameters entropy $(\mathrm{H})$, anisotropy $(\mathrm{A})$, and averaged alpha angle $(\bar{\alpha})[23]$ are shown in Fig. 8 for the detailed area of the second acquisition presented in Fig. 5, for a pruning threshold $\delta_{p}=-3 \mathrm{~dB}$. It compares the values obtained by both BPT approaches and also the $7 \times 7$ multilook (ML) filtering as a reference, which corresponds to the maximum likelihood estimator [24]. A qualitative comparison of the results reveals that similar values are obtained by all the methods. However, the BPT approaches may obtain a better noise reduction due to employing a larger number of samples for polarimetric response estimation while also preserving the contours. This 


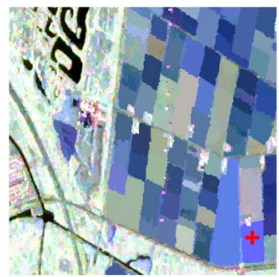

(a)

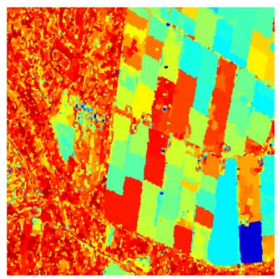

(d)

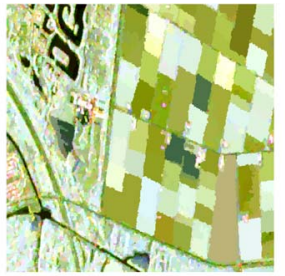

(b)

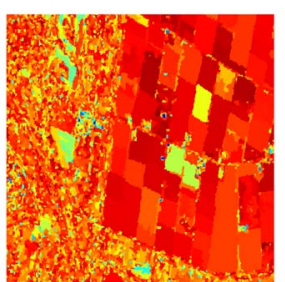

(e)

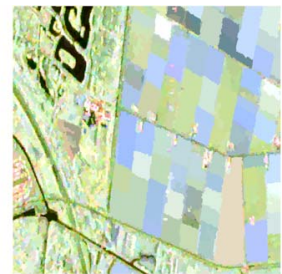

(c)

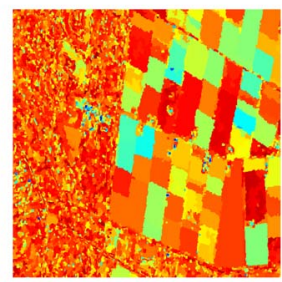

(f)
Fig. 9. Pauli and entropy $H$ images for different acquisitions processed with the TE BPT. The data set has been processed with $\delta_{p}=-3 \mathrm{~dB}$ and employing $N=8$. (a) First acquisition. (b) Fourth acquisition. (c) Eighth acquisition. (d) $\mathrm{H}$, first acquisition. (e) $\mathrm{H}$, fourth acquisition. (f) $\mathrm{H}$, eighth acquisition.

effect is more clear over the TE BPT since it can benefit from increased region differentiation over temporally fixed contours, as stated before.

In order to see the temporal evolution of this information and its preservation, Fig. 9 shows the Pauli representation of an area of the data set for the first, fourth, and eighth acquisitions processed with the TE BPT with a pruning threshold of $\delta_{p}=-3 \mathrm{~dB}$ and the obtained entropy $H$ parameter. Note that, on the first acquisition, most of the agricultural fields are not grown; on the fourth acquisition, they are fully grown; and, finally, on the eighth acquisition, most of them have already been harvested. This temporal evolution may be seen in the Pauli images in Fig. 9(a)-(c). When the plants are not grown, the surface scattering dominates, appearing as blue in the Pauli representation, but when the plants are fully grown, the volume scattering dominates, represented in green color. Consequently, the polarimetric response of these areas changes substantially over the different acquisitions, and so does the entropy parameter, as can be seen in Fig. 9(d)-(f), ranging from low entropy when there is surface scattering to high entropy when the volume scattering dominates.

Fig. 10 shows the temporal evolution of the $\mathrm{H} / \mathrm{A} / \bar{\alpha}$ parameters for the field marked with a red cross in Fig. 9(a) among all the acquisitions. The evolution is shown for the two presented BPT approaches, employing in both cases $\delta_{p}=-3 \mathrm{~dB}$, and for the $7 \times 7 \mathrm{ML}$ filtering, as a reference. Note that the cross shown in Fig. 9(a) is far enough from the field edges in order to ensure that the ML filter window is covering homogeneous pixels. It may be seen that very similar trends are obtained by both BPT processing methods, which are also very similar to the evolution obtained when employing the ML filtering, which means that the proposed BPT-based processing preserves also the polarimetric temporal evolution of the data. Moreover, the proposed methods may employ a much larger number of homogeneous samples with respect to the ML filtering, resulting in a better estimation of the polarimetric response and evolution while also having a better spatial resolution preservation, maintaining the region contours and small details of the scene. Note that the
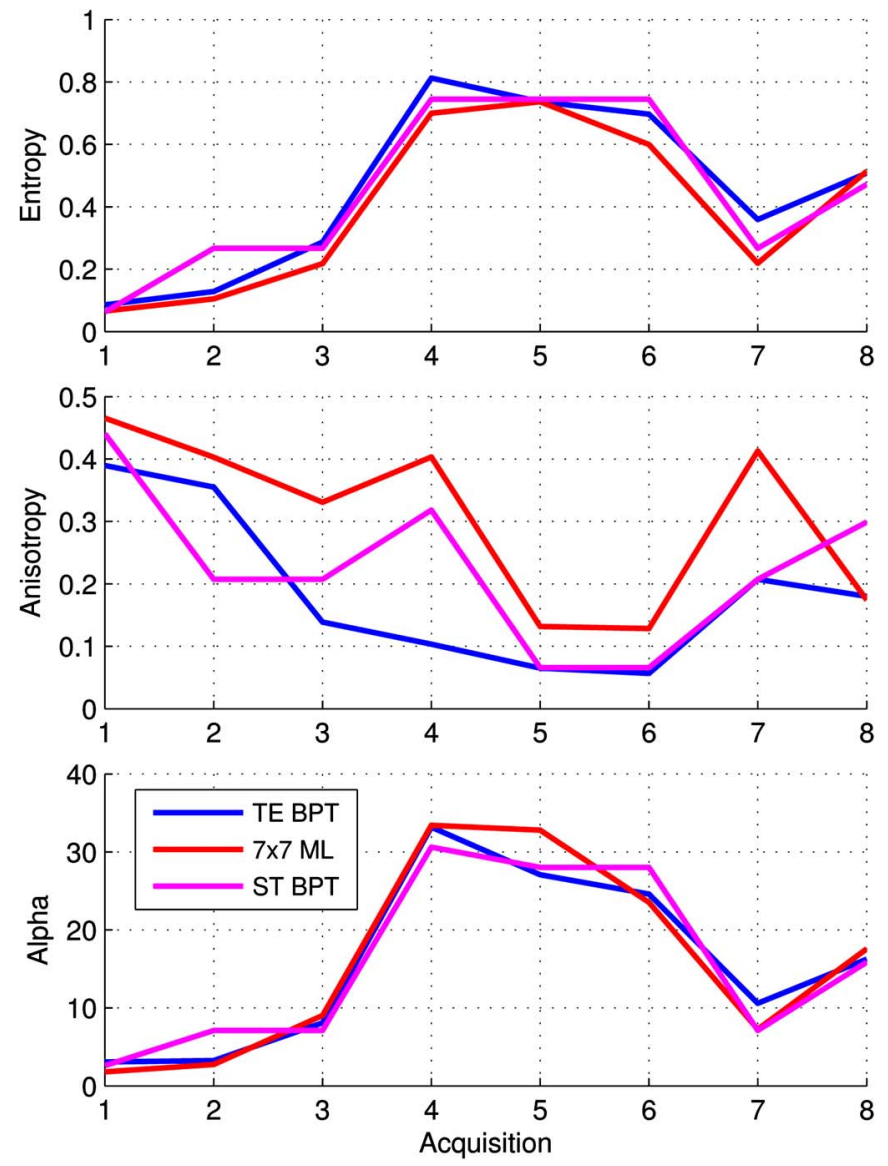

Fig. 10. Evolution of the $\mathrm{H} / \mathrm{A} / \bar{\alpha}$ parameters for the agricultural field marked in Fig. 9(a). The full data set has been processed with the TE BPT, with a $7 \times$ $7 \mathrm{ML}$ filter, and with the ST BPT. For the BPT pruning, $\delta_{p}=-3 \mathrm{~dB}$ has been employed on both cases.

TABLE II

Observed Execution TIMES For DifFERENT PROCESSING Stages of THE PROPOSED TECHNIQUES

\begin{tabular}{|c|c|c|c|}
\hline Processing & $\begin{array}{c}\text { Flevoland } \\
\text { ST BPT }\end{array}$ & $\begin{array}{c}\text { Flevoland } \\
\text { TE BPT }\end{array}$ & $\begin{array}{c}\text { Barcelona } \\
\text { TE BPT }\end{array}$ \\
\hline \hline BPT Construction & $5 \mathrm{~h} 24 \mathrm{~m}$ & $37 \mathrm{~m} \mathrm{29s}$ & $5 \mathrm{~h} 34 \mathrm{~m}$ \\
\hline Prune $\delta_{p}=-2 d B$ & $0.24 \mathrm{~s}$ & $0.52 \mathrm{~s}$ & $6.2 \mathrm{~s}$ \\
\hline Prune $\delta_{p}=-1 d B$ & $0.04 \mathrm{~s}$ & $0.19 \mathrm{~s}$ & $3.4 \mathrm{~s}$ \\
\hline Prune $\delta_{p}=0 d B$ & $0.02 \mathrm{~s}$ & $0.08 \mathrm{~s}$ & $2.3 \mathrm{~s}$ \\
\hline
\end{tabular}

estimation of the $\mathrm{H} / \mathrm{A} / \bar{\alpha}$ parameters employing a finite number of samples is biased, as stated in [25]. As a consequence, the entropy parameter is underestimated, whereas the anisotropy parameter is overestimated. As it may be seen, the entropy obtained by the ML filtering is below the one obtained by the BPT while the anisotropy is above it. The BPT is employing a much larger number of samples, thus reducing the bias with respect to the ML.

To give an idea of the complexity of the technique in practice, Table II shows the observed execution times of different processing stages of the BPT-based processing. A processing server has been employed for computation, having 12 Intel Xeon processors. As stated in Section II, the most timeconsuming part is the BPT construction, due mainly to the similarity measure computation. Note, however, that this stage has to be performed only once per data set. When the BPT 


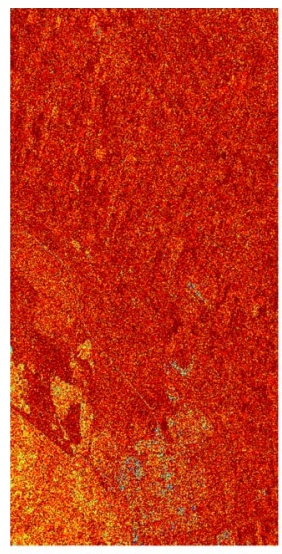

(a)

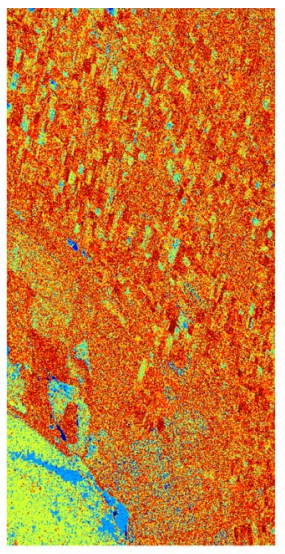

(b)

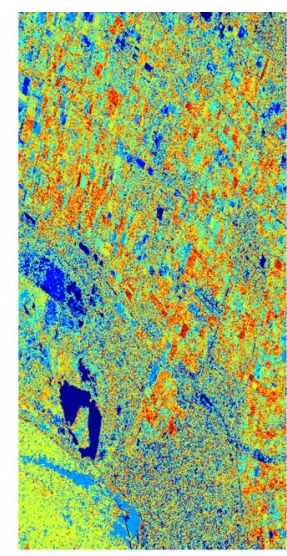

(c)
Fig. 11. Temporal changes for different pruning factors $\delta_{p}$. "No changes" is represented in blue, and "seven changes" is represented in red. (a) $\delta_{p}=-5 \mathrm{~dB}$. (b) $\delta_{p}=-3 \mathrm{~dB}$. (c) $\delta_{p}=-1 \mathrm{~dB}$.

has been constructed, many different pruning processes may be performed to generate results for distinct applications at many scales, and in fact, this pruning process is really fast, as it may be observed from Table II. It may also be seen that the ST BPT has a larger computational complexity than the TE BPT since its number of nodes gets increased by a factor of $N$. On the other hand, the pruning process may be performed faster on the ST BPT due to the simpler computation of the pruning criterion.

\section{TEMPORAL INFORMATION ANALYSIS}

In Section VI, the results are shown after processing a real data set for the speckle filtering or target response estimation application. However, temporal series data sets enable the analysis and exploitation of the additional time dimension in order to analyze the evolution of the scene across the different acquisition times. In this section, two additional applications are outlined in order to analyze the temporal information after BPT pruning: temporal change analysis and temporal stability.

As stated before, the ST BPT contains regions with arbitrary shapes in the space-time domain, which are composed of pixels of different acquisitions having similar polarimetric response. The change analysis application naturally arises when examining the region contours of this data representation in the temporal dimension, as proposed in [19]. A map may be generated indicating, for each spatial pixel of the scene, the number of contours in the temporal dimension, i.e., the number of times that the portion of the scene represented by this spatial pixel changes its assigned region among all the acquisitions, and consequently, it changes its polarimetric response. Then, the values of this map range from 0 changes, when the spatial pixel belongs to the same region and there is no change in the polarimetric response on all the acquisitions, to $N-1$, when there is a region change on every acquisition.

Fig. 11 shows the maps of the number of temporal contours for each spatial pixel of the scene. Since the Flevoland data set is composed of eight acquisitions, the map values range from zero, represented in blue, to seven changes, corresponding to red. As it may be seen, when the pruning threshold $\delta_{p}$ gets increased, a lower number of temporal contours are detected.
This is caused by the fact that bigger regions are obtained also in the temporal dimension when $\delta_{p}$ is increased, resulting in fewer temporal contours. Note that the BPT construction process defined in Section IV-A and the BPT pruning defined in Section $\mathrm{V}$ are sensitive to the full polarimetric information, under the Gaussian hypothesis, and consequently, these maps are also sensitive to this information. These maps give an idea of the number of polarimetric changes for each spatial pixel over time. It may be seen that less temporal contours are obtained over closed waters or small stable structures within urban areas, whereas larger number of changes may be observed over agricultural fields. However, although these maps give an idea of the number of temporal changes, they do not indicate the relevance of these changes. For instance, although a small number of changes may be seen on urban areas, maybe some of them are larger than the ones produced over the sea. Additionally, the results shown in Fig. 11 seem to be considerably noisy, as the obtained regions may have arbitrary shapes over time, which may result in different number of temporal contours for spatial pixels corresponding to the same agricultural field. Results giving an idea of the amount of change for each field or structure of the scene could be more useful. In order to have such results, a temporal analysis should be performed over the TE BPT since it provides spatially fixed contours, as shown in Fig. 7.

The TE BPT contains the temporal information within the region model, but since it performs a spatial segmentation of the data, it does not have temporal contours, and a completely different analysis has to be defined. The $\mathbf{Z}_{e}$ region model contains the polarimetric response temporal evolution for the region, having each acquisition response within the different $\mathbf{Z}_{i i}$ matrices, as stated before. Therefore, a new temporal stability measure is defined based on analyzing the amount of change between all the different $\mathbf{Z}_{i i}$ matrices of each region. In order to be consistent with the BPT construction and processing, a similarity measure is also employed to evaluate the similarity $t_{s}$ between the different polarimetric matrices over all the acquisitions

$$
t_{s}=\frac{2}{N(N-1)} \sum_{i=1}^{N} \sum_{j=i+1}^{N}\left\|\log \left(\mathbf{Z}_{i i}^{-1 / 2} \mathbf{Z}_{j j} \mathbf{Z}_{i i}^{-1 / 2}\right)\right\|_{F} .
$$

Note that the $t_{s}$ measure defined in (9) corresponds to the average geodesic similarity measure between all the acquisitions. By contrast to the number of temporal contours, the $t_{s}$ measure is not mathematically bounded, ranging from zero, when there is no change on the polarimetric response over all the acquisitions, to infinity, when there are big changes over the acquisitions. With real data, since the measured values are bounded, $t_{s}$ ranges approximately in the interval $[0,6]$, but almost all the values are concentrated within the range $[0,3]$.

Fig. 12 shows the $t_{s}$ measure over the Flevoland data set for different values of the pruning threshold $\delta_{p}$. As it may be seen, for lower values of $\delta_{p}$, the temporal stability measure obtains larger values, due to the smaller amount of filtering, resulting in larger variability of the obtained values, whereas as the pruning factor increases, larger regions are obtained, resulting in larger filtering and variability reduction. Analyzing 


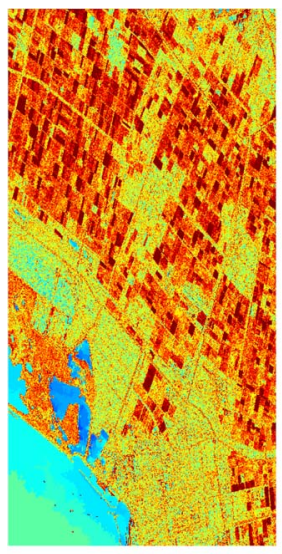

(a)

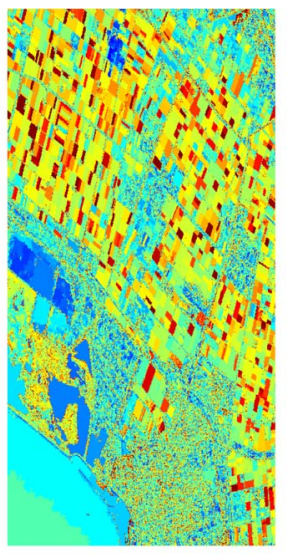

(b)

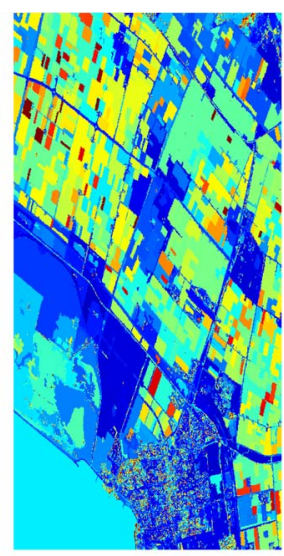

(c)
Fig. 12. Temporal stability measure $t_{s}$ for different pruning factors $\delta_{p} . t s=0$ is represented in blue, and $t_{s}=2.5$ is represented in red. (a) $t_{s}, \delta_{p}=-5 \mathrm{~dB}$. (b) $t_{s}, \delta_{p}=-3 \mathrm{~dB}$. (c) $t_{s}, \delta_{p}=-1 \mathrm{~dB}$.

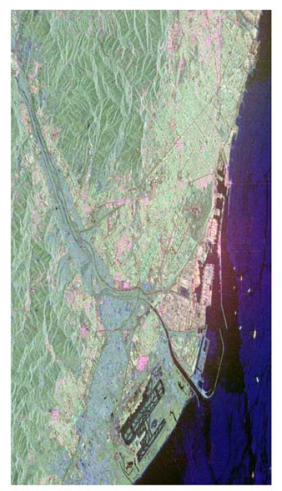

(a)

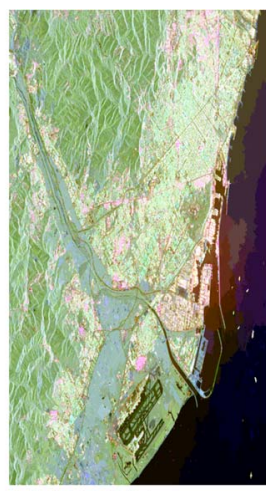

(b)

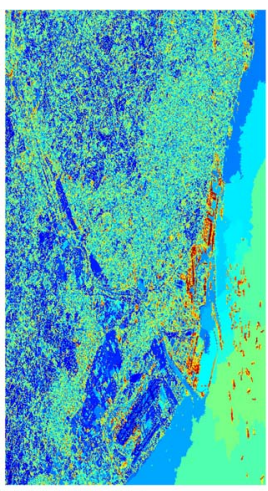

(c)
Fig. 13. (a) Pauli of the first acquisition of the Barcelona data set, (b) first acquisition processed with the TE BPT, and (c) temporal stability measure $t_{s}$ for $\delta_{p}=-2 \mathrm{~dB}$. $t s=0$ is represented in blue, and $t_{s}=3$ is represented in red. (a) Original first acquisition. (b) $\delta_{p}=-2 \mathrm{~dB}$. (c) $t_{s}, \delta_{p}=-2 \mathrm{~dB}$.

the $t_{s}$ results, it may be seen that larger variability in terms of the polarimetric temporal evolution is produced over the agricultural fields, whereas closed waters, for instance, have a more stable polarimetric response. These results are, in fact, similar to the ones obtained by the number of changes in the temporal dimension, shown in Fig. 11; however, both results represent different information. The temporal contours give an idea of the number of changes in the polarimetric response over time, whereas $t_{s}$ measures the amount of temporal polarimetric change for each region.

In order to analyze the ability of the temporal stability measure to capture important changes in the scene, a new data set is employed, containing 35 RADARSAT-2 Fine Quad-Pol images of the city of Barcelona in Spain. All the images where acquired with the FQ9 beam, from January 20, 2010 to May 9, 2012, with an acquisition every 24 days. Fig. 13(a) shows the Pauli representation of the first acquisition of the Barcelona data set, Fig. 13(b) shows the first acquisition of the processed data set employing the TE BPT with $\delta_{p}=-2 \mathrm{~dB}$, and Fig. 13(c) shows the $t_{s}$ measure over the data set. By contrast to the Flevoland data set, this data set contains mainly urban and industrial areas.

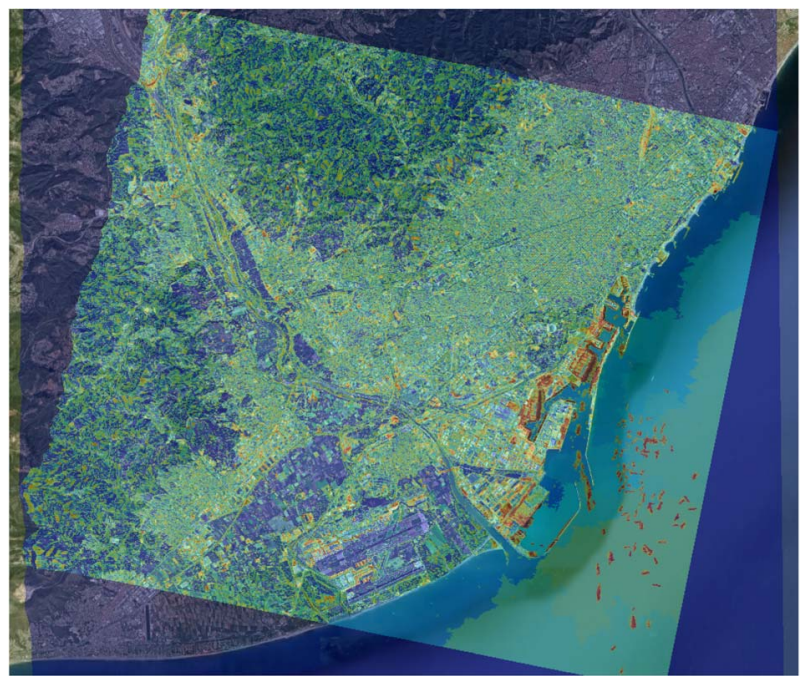

Fig. 14. Geocoded $t_{s}$ measure for the Barcelona data set as an overlay with an optical image [26].

In order to easily identify the different regions of the processed data set over the scene, the $t_{s}$ results shown in Fig. 13(c) have been geocoded, and they have been used as an overlay with an optical image from [26], with a certain degree of transparency, as shown in Fig. 14. As it can be seen, several red spots appear over the sea, at the lower right part of Fig. 14, which correspond to the different vessels that were present at different acquisitions. Note that these vessels, since they are present only in one acquisition, represent a significant change in terms of the polarimetric response with respect to the underlying sea, and consequently, the $t_{s}$ measure indicates a large value, appearing in red.

Over the land area, some zones appear in red, indicating large changes, particularly over the harbor area. A zoom of the results shown in Fig. 14 corresponding to an area of the Barcelona harbor is shown in Fig. 15(a). In the center of the image, large changes are detected over a container area, which is seen on the ground photography in Fig. 15(b) [27]. These container areas are completely different from acquisition to acquisition since containers are constantly stacked and unstacked from the ships. This task is performed by big cranes located near the loading bay, which may be seen in Fig. 15(c) [27], which are placed over rails, and consequently, their position changes over different acquisitions. In Fig. 15(a), big spots may be seen around the shore, corresponding to these cranes and also to the fact that different ships are docked at the loading bays on some acquisitions.

Another area of the scene having large changes is the Barcelona airport zone. A zoom of the results corresponding to the Terminal-1 airport area is shown in Fig. 16(a). At the bottom part of the image, the Terminal-1 building may be seen, having some fingers connected to it through the different boarding gates. Small dots may be seen over the fingers since they are moving every time that they are attached to a plane. Additionally, on zones where planes where placed at some acquisitions, big changes are also detected, appearing in yellow and red colors. At the top part of the image, some plane parking lots may be clearly identified as they are zones with high 


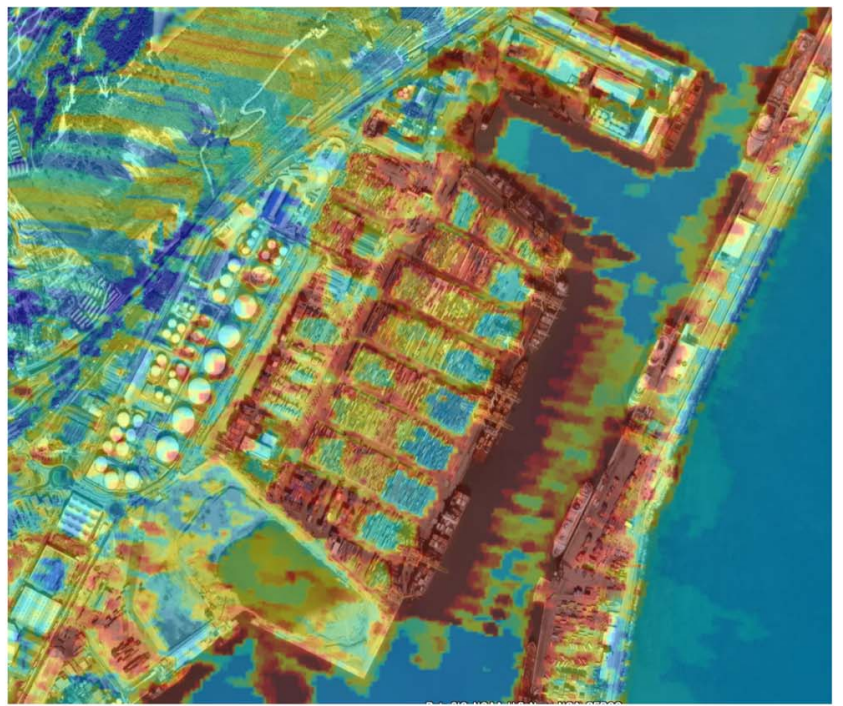

(a)

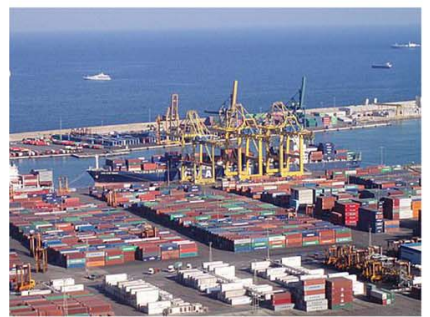

(b)

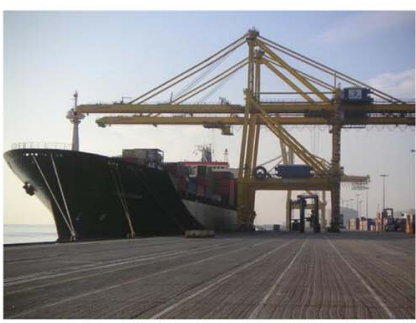

(c)
Fig. 15. (a) Geocoded $t_{s}$ measure over the Barcelona harbor [26]. (b) Container area with (c) a ship loading bay and cranes may be seen [27].

probability of containing different planes from acquisition to acquisition.

Fig. 16(b) shows another part of the Barcelona airport corresponding to the hangar area. At the bottom left part of the image, some parking lots can be observed. However, the most important change detected, in the top right part of the image, corresponds to a big hangar that has been constructed during the acquisition campaign. Fig. 16(c) and (d) show the photographs of the hangar in construction and finished, respectively. Note that, in the optical image employed in Fig. 16(b), it was under construction. These results show the ability of the proposed BPT-based processing technique and the temporal stability measure $t_{s}$ to capture scene changes produced by building activities.

Moreover, the extended model $\mathbf{Z}_{e}$ contains precise information about the polarimetric temporal evolution of each scene region within the $\mathbf{Z}_{i i}$ matrices, which may be employed to characterize the different detected changes. As an example, Fig. 17 shows the evolution of the entropy $(H)$ and averaged alpha $(\bar{\alpha})$ parameters for the different acquisitions. It may be seen that, during the construction process, they change considerably due to the different building tasks but, at some point, around the 14th acquisition, $H$ and $\bar{\alpha}$ parameters become stable, presumably when the construction process has finished. Note that, in this case, the entropy becomes very low and the averaged alpha angle presents a low value, indicating surface scattering probably caused by the roof of the hangar.

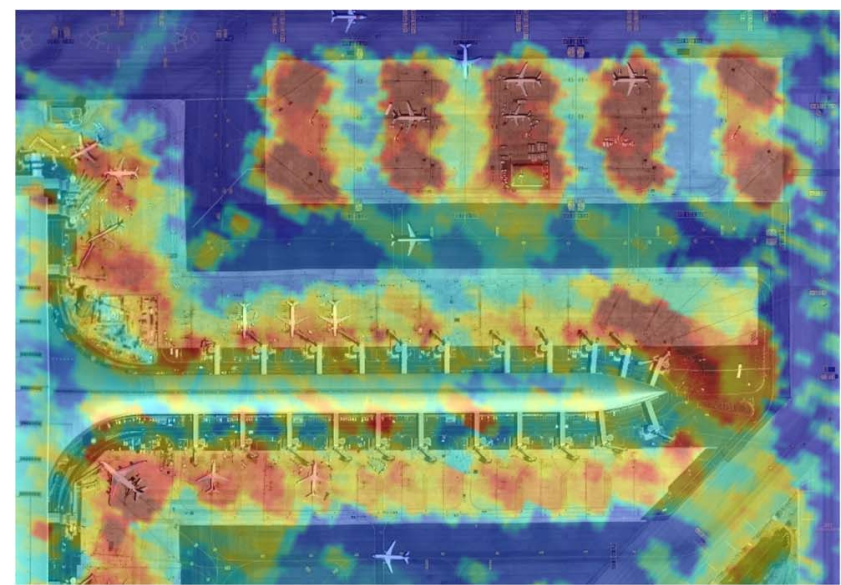

(a)

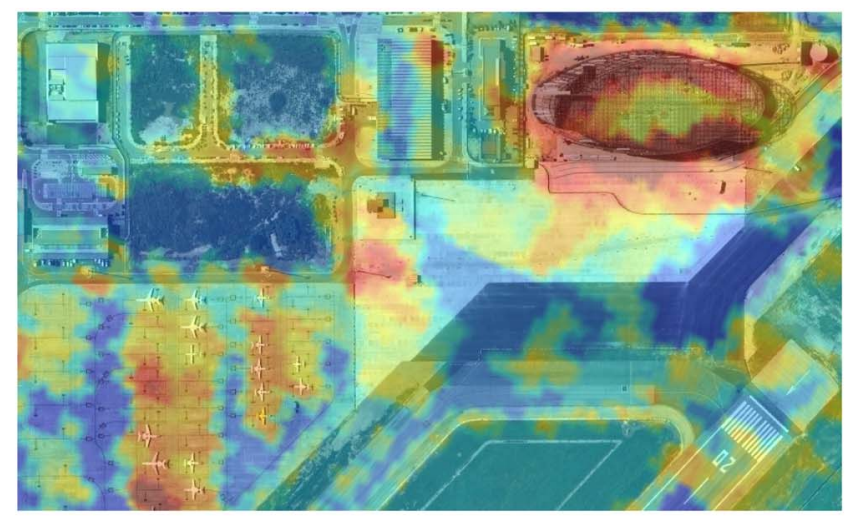

(b)

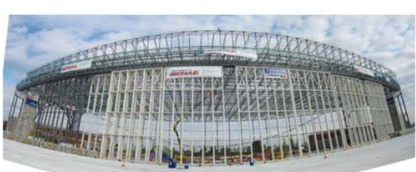

(c)

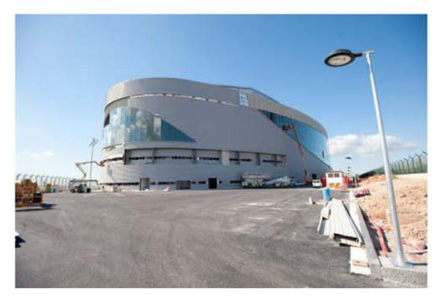

(d)
Fig. 16. Geocoded $t_{s}$ measure over (a) the Barcelona airport Terminal-1 area and (b) the hangar area [26]. Hangar (c) under construction and (d) after construction finished [27].

In order to see the ability of the proposed method to detect very localized changes of the scene, Fig. 18 shows the $t_{s}$ results over the Montjuic fountain area in Barcelona. A small red dot may be seen at the center of the image, corresponding to the building in this area of a monument consisting of four columns during the data set acquisition period of time. Fig. 18(b) and (c) shows the photographies of this area before and after the construction of this monument. This monument is, in fact, much smaller than the hangar shown in Fig. 16 and not much larger than the spatial resolution of the sensor, but due to the ability of the BPT representation to preserve small details of the data, this scene change may be clearly identified.

To compare the proposed method with a state-of-the-art polarimetric change detection technique, a modification of the log likelihood-ratio test statistic measure defined in [28] is proposed. Note that the change measure $\ln Q$ defined in [28] is intended to determine the change between two different 


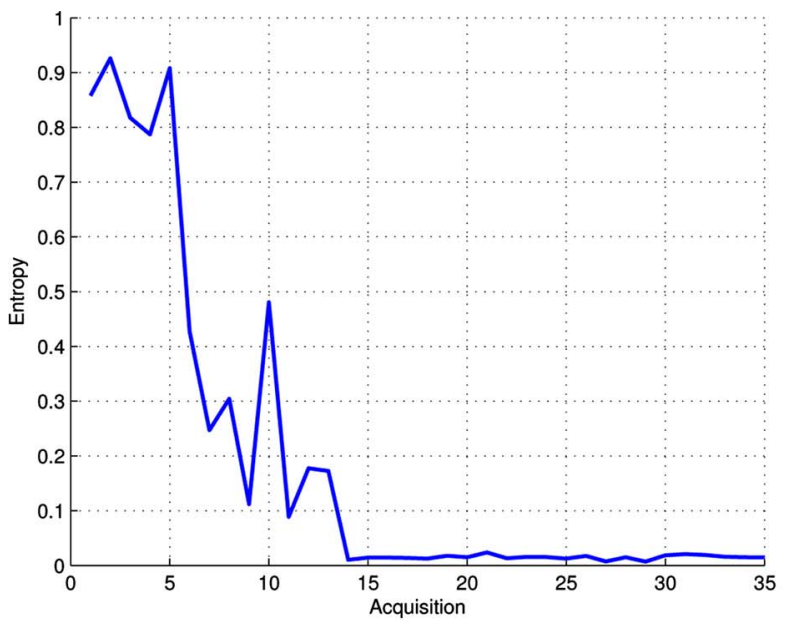

(a)

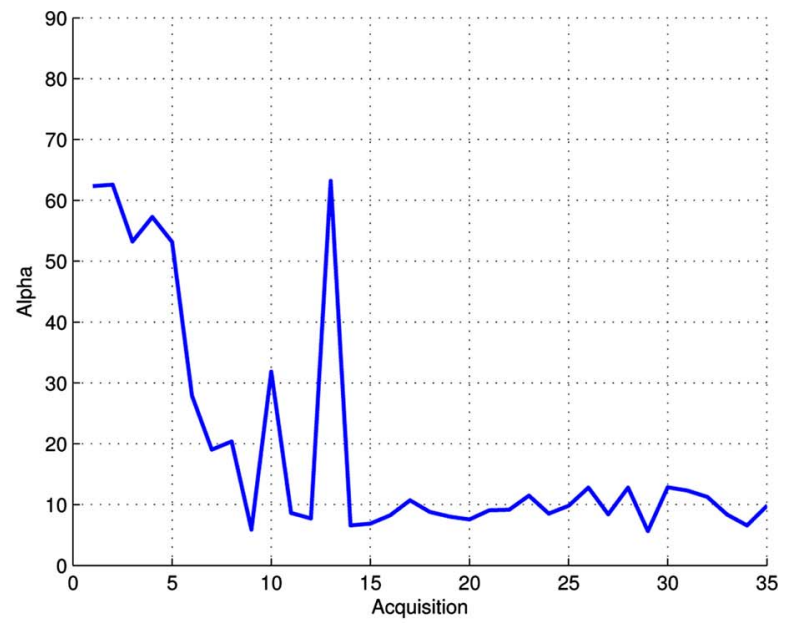

(b)

Fig. 17. (a) Entropy and (b) the averaged alpha angle temporal evolution for the hangar over different acquisitions. (a) $H$ evolution for the hangar. (b) $\bar{\alpha}$ evolution for the hangar.

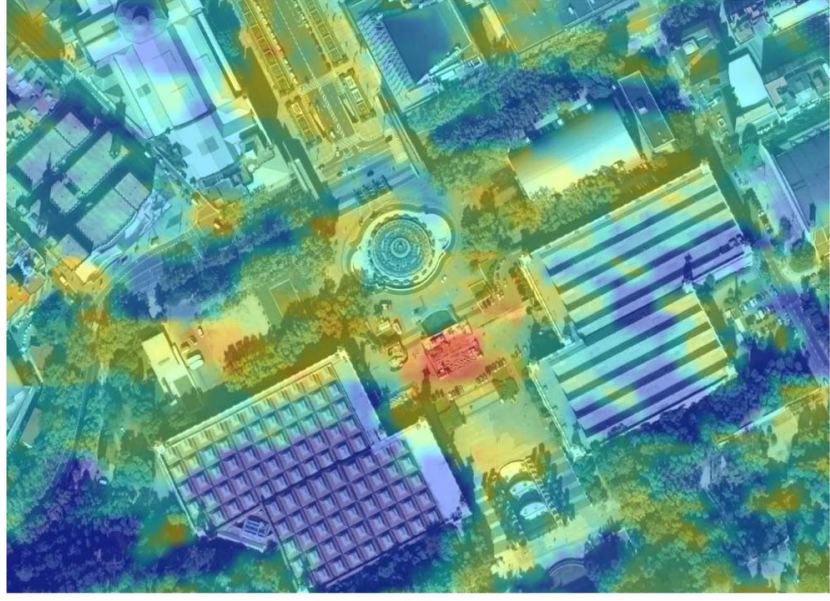

(a)

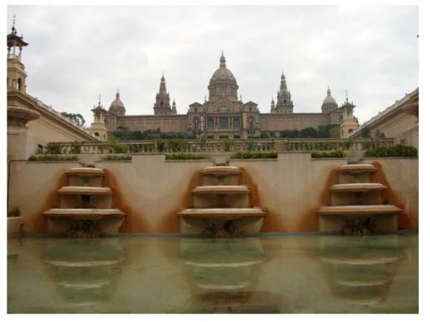

(b)

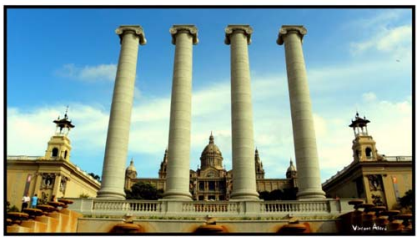

(c)
Fig. 18. (a) Geocoded $t_{s}$ measure over the Montjuic area in Barcelona [26]. Photographs (b) before and (c) after the construction of the four-column monument [27].

acquisitions. When dealing with PolSAR time series, this measure has to be extended to $N$ different acquisitions. Adapting the test statistic defined in [28] according to [24], we have defined the extended log likelihood-ratio test statistic measure

$$
-\ln Q_{e}=-n_{l}\left(\sum_{i=1}^{N} \ln \left|\mathbf{Z}_{i i}\right|-N \ln \left|\mathbf{Z}_{s}\right|+p N \ln N\right)
$$

where $n_{l}$ represents the number of looks, $\left|\mathbf{Z}_{i i}\right|$ represents the determinant of the $p \times p$ matrix $\mathbf{Z}_{i i}$, and $\mathbf{Z}_{s}=\sum_{i=1}^{N} \mathbf{Z}_{i i}$.

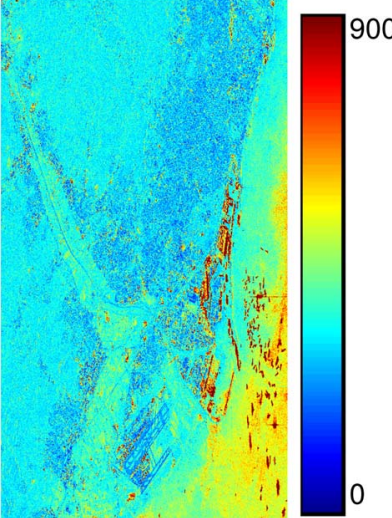

(a)

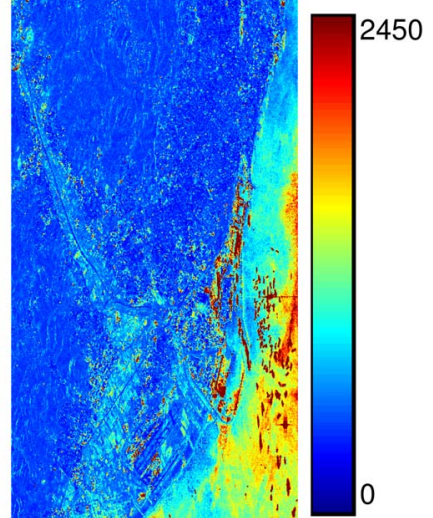

(b)
Fig. 19. $\quad-\ln Q_{e}$ test statistic results for change detection over the Barcelona data set. (a) $-\ln Q_{e}, 3 \times 3 \mathrm{ML}$. (b) $-\ln Q_{e}, 7 \times 7 \mathrm{ML}$.

The $-\ln Q_{e}$ measure ranges from 0 when $Q_{e}=1$, indicating no change, to $\infty$ when $Q_{e}=0$, indicating a strong change. Note that this measure requires full-rank matrices in order to compute the determinant. This implies that some initial filtering must be applied to the data. In the following, we will consider an initial ML filter in order to apply (10). The amount of initial filtering plays an important role in the obtained results. A small amount of filtering will produce noisy results, whereas a large filtering will reduce the spatial resolution. Fig. 19 shows the results of applying (10) after a $3 \times 3$ and a $7 \times 7$ ML filter over the Barcelona data set.

As it may be seen, the contrast increases when the amount of filtering gets increased. For instance, the contour between land and sea areas is more clear in Fig. 19(b) than in Fig. 19(a). To see more clearly the differences in terms of the initial filtering, a geocoded crop of the airport area is shown in Fig. 20(a)-(c) as an overlay with an optical image [26]. As stated before, increasing the amount of initial filtering increases the contrast between change and no-change areas. However, it may be seen that it also implies a resolution loss, as the areas of the parking lots get enlarged according to the filter window size. 


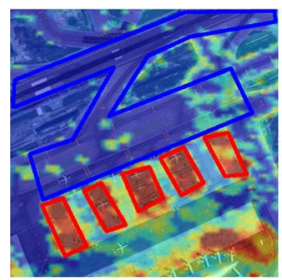

(a)

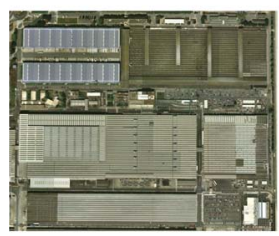

(d)

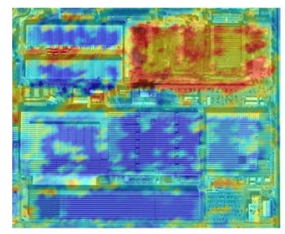

(g)

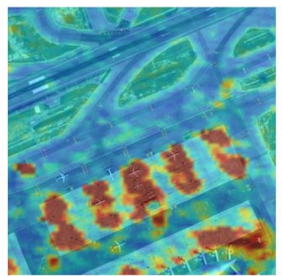

(b)

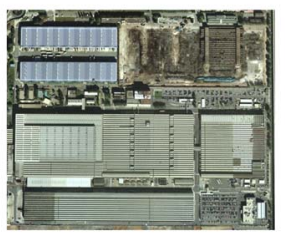

(e)

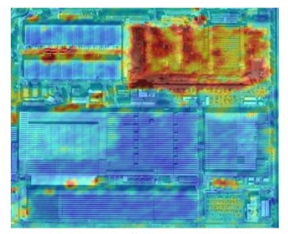

(h)

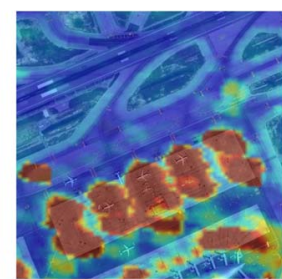

(c)

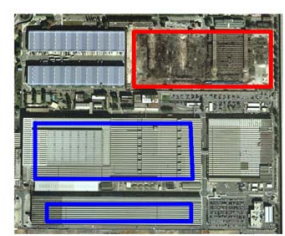

(f)

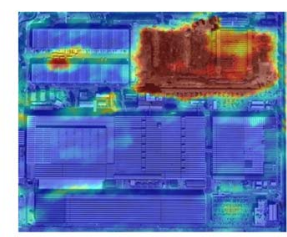

(i)
Fig. 20. Geocoded $t_{s}$ and $-\ln Q_{e}$ comparison over the airport and a building area in the Barcelona harbor as an overlay with an optical image [26]. (a) $t_{s}$, $\delta_{p}=-2$ dB. (b) $-\ln Q_{e}, 3 \times 3$ ML. (c) $-\ln Q_{e}, 7 \times 7$ ML. (d) Before. (e) After. (f) Zones for $S$ test. (g) $t_{s}, \delta_{p}=-2 \mathrm{~dB}$. (h) $-\ln Q_{e}, 3 \times 3 \mathrm{ML}$. (i) $-\ln Q_{e}, 7 \times 7 \mathrm{ML}$

The $t_{s}$ and $-\ln Q_{e}$ measures do not have the same range of values. Moreover, the $-\ln Q_{e}$ value strongly depends on the amount of filtering. Consequently, to assess quantitatively the separability between change and no-change areas, the $S$ measure will be employed

$$
S=\frac{\sigma_{c}+\sigma_{n c}}{\left|\mu_{c}-\mu_{n c}\right|}
$$

where $\sigma_{c}, \sigma_{n c}$ and $\mu_{c}, \mu_{n c}$ stand for the standard deviation and the mean of the change and no-change areas, respectively. Consequently, a lower value of $S$ indicates that both classes can be better separated by the change measure. To evaluate (11), two areas of the Barcelona data set have been analyzed in detail, shown in Fig. 20, corresponding to the Barcelona airport and to some industrial buildings on the harbor, respectively. The airport area shown in Fig. 20(a) has some relatively small change areas, in terms of the spatial resolution, whereas Fig. 20(f) contains a large change corresponding to an industrial building that has been demolished during the acquisition campaign. This change may be seen in optical images between Fig. 20(d) and (e) [26]. To evaluate the $S$ measure, some areas have been manually marked in Fig. 20(a) and (f) corresponding to change and no change, marked with red and blue color, respectively. Tables III and IV show the values of $\sigma_{c}, \sigma_{n c}, \mu_{c}$, and $\mu_{n c}$ and the separation measure $S$ over the areas shown in Fig. 20(a) and (f) for the $t_{s}$ measure with two different pruning thresholds and for the $-\ln Q_{e}$ measure with two different initial filtering.

As it may be seen in Table III, for the airport area, having small change zones, the separation of the BPT proposed method is better in both cases. Moreover, for the $-\ln Q_{e}$ measure, an increase of the initial filtering produces a worse separation as the resolution loss and mixture of areas becomes more signif-
TABLE III

SEPARABILITy BetweEn CHANGE AND No-CHANGE AREAS OF THE AIRPORT SHOWN IN Fig. 20(a)

\begin{tabular}{|c|c|c|c|c|c|}
\hline Measure & $\mu_{c}$ & $\mu_{n c}$ & $\sigma_{c}$ & $\sigma_{n c}$ & $S$ \\
\hline \hline$t_{s}, \delta_{p}=-2 d B$ & 3.034 & 0.4207 & 0.7578 & 0.3785 & 0.4348 \\
\hline$t_{s}, \delta_{p}=-1 d B$ & 2.993 & 0.2944 & 0.8023 & 0.1900 & 0.3678 \\
\hline$-\ln Q_{e}, 3 \times 3 \mathrm{ML}$ & 1095 & 259.1 & 462.0 & 52.87 & 0.6157 \\
\hline$-\ln Q_{e}, 7 \times 7 \mathrm{ML}$ & 4478 & 489.7 & 2179 & 460.1 & 0.6615 \\
\hline
\end{tabular}

TABLE IV

Separability Between Change AND No-Change AREAS FOR THE Barcelona Harbor Industrial AREA SHOWN IN Fig. 20(f)

\begin{tabular}{|c|c|c|c|c|c|}
\hline Measure & $\mu_{c}$ & $\mu_{n c}$ & $\sigma_{c}$ & $\sigma_{n c}$ & $S$ \\
\hline \hline$t_{s}, \delta_{p}=-2 d B$ & 2.222 & 0.6224 & 0.2920 & 0.3408 & 0.3956 \\
\hline$t_{s}, \delta_{p}=-1 d B$ & 2.077 & 0.4459 & 0.2760 & 0.1832 & 0.2816 \\
\hline$-\ln Q_{e}, 3 \times 3 \mathrm{ML}$ & 710.8 & 209.0 & 145.6 & 62.23 & 0.4143 \\
\hline$-\ln Q_{e}, 7 \times 7 \mathrm{ML}$ & 2611 & 279.2 & 643.5 & 82.27 & 0.3112 \\
\hline
\end{tabular}

icant. On the contrary, the BPT approach is able to preserve the spatial resolution even when the amount of filtering gets increased, for $\delta_{p}=-1 \mathrm{~dB}$, producing better results of the $S$ measure. For the industrial building area, shown in Table IV, the resolution loss due to increasing the amount of initial filtering is not as significant as in the previous case, producing an increase of the separability due to the increase of contrast for the $-\ln Q_{e}$ measure with a $7 \times 7 \mathrm{ML}$. In this scenario, the BPT also obtains better results in terms of the separability measure $S$ for low and high filtering, i.e., $t_{s}$ with $\delta_{p}=-2 \mathrm{~dB}$ versus $-\ln Q_{e}$ with $3 \times 3 \mathrm{ML}$ and $t_{s}$ with $\delta_{p}=-1 \mathrm{~dB}$ versus $-\ln Q_{e}$ with a $7 \times 7 \mathrm{ML}$, respectively. The improvement of the BPT-based technique is produced in this case by a combination of a better spatial resolution preservation and a larger amount of filtering over large homogeneous areas.

\section{CONCLUSION}

In this paper, the processing of temporal series of PolSAR images has been addressed employing the BPT data abstraction. Temporal information and its dynamics may be addressed from different points of view. In this paper, two different alternatives have been considered. On the one hand, it may be assumed that a target is characterized by its PolSAR radar response. As a consequence, samples from different acquisitions may be combined while there is no substantial changes on their polarimetric response. This approach involves a 3-D space-time processing, leading to the ST BPT. On the other hand, it may be assumed that a target is characterized by its temporal PolSAR response evolution. In this case, the temporal dimension is included within the signal model, and the goal is to combine pixels of the scene presenting the same temporal evolution among the acquisitions. This approach leads to the TE BPT. The ST BPT may achieve a better estimation of the polarimetric response by means of combining samples of different acquisitions, and due to its versatility, it may adapt to region contours changing over time. However, due to the different acquisition information mixture, it prevents the exploitation of the interferometric information. The TE BPT may achieve a better detection of the temporally fixed spatial contours since a region differentiation enhancement is produced when taking into account the temporal evolution. Nonetheless, since it is a 2-D segmentation, it cannot adapt properly to the spatial contours that are changing over time. Additionally, it has the advantage 
of being able to exploit the interferometric information since it may be included within the region model.

From the aforementioned conclusions, we can deduce that the ST BPT may be a good option for areas where almost no information can be extracted from the interferometric information, which may be produced by a strong temporal decorrelation, or where the contours are not spatially fixed as, for instance, the sea or ocean areas. On the other hand, the increase of region differentiation over fixed contours of the TE BPT makes it a good option for processing the land areas, where most of the contours are fixed over time. Moreover, the regions obtained by this representation uniquely correspond to spatial areas of the scene, making their interpretation and analysis easier. Note then that the way to process the temporal dimension depends on target polarimetric temporal behavior and characterization, and nowadays, few studies have addressed this issue [29].

In order to exploit these data representations, a homogeneitybased tree pruning has been proposed. The biggest homogeneous regions from the tree are extracted. A region homogeneity measure is defined, sensitive to the complete polarimetric information, under the Gaussian hypothesis, taking into account the temporal dimension. The proposed BPT pruning strategy naturally leads to a time series speckle filter or polarimetric response estimator. Under the point of view of these applications, the proposed technique is able to obtain large regions, representing homogeneous areas of the scene, while also preserving small details and maintaining the resolution of the original data. Moreover, it has been shown that the polarimetric information of the data is preserved by both approaches, without introducing any bias or distortion.

In addition, a temporal analysis of the results has been proposed, in order to analyze the variability of the scene over time. The scene changes are directly identified by the ST BPT as region temporal contours. Then, a scene map has been generated, indicating the number of changes for each pixel. However, these results do not give a clear idea of the significance of the changes, and they seem noisy, due to the arbitrary shape of the regions in the space-time domain. To solve these issues, a temporal stability measure has been defined over the TE BPT. This measure gives a clear idea of the relevance of the changes of each region among all the acquisitions, and additionally, it may benefit from the increase of region differentiation of the TE BPT over temporally fixed contours. This approach has proven to be sensitive to human-made scene changes, including building construction and transportation, for instance, over the harbor and the airport of the Barcelona city data set. Moreover, the ability of the BPT to preserve small details of the data has been useful for this application to identify very localized changes of the scene. Additionally, an extension of a stateof-the-art change detection technique based on a test statistic on the complex Wishart distribution has been proposed, and it has been compared with the temporal stability measure. In this comparison, the benefits of the region-based BPT approach have been shown in terms of a better separability between change and no-change areas. Finally, it is worth to notice that this technique allows the characterization of the target temporal dynamics, providing the capability to analyze or interpret the detected changes.

\section{REFERENCES}

[1] J. S. Lee and E. Pottier, Polarimetric Radar Imaging: From Basics to Applications. Boca Raton, FL, USA: CRC Press, 2009.

[2] S. Cloude, Polarisation: Applications in Remote Sensing. New York, NY, USA: Oxford Univ. Press, 2009.

[3] C. Elachi and J. J. van Zyl, Introduction To The Physics and Techniques of Remote Sensing. Hoboken, NJ, USA: Wiley, 2006, ser. Wiley Series in Remote Sensing and Image Processing.

[4] F. T. Ulaby and C. Elachi, Radar Polarimetry for Geoscience Applications. Norwood, MA, USA: Artech House, 1990, ser. Artech House Remote Sensing Library.

[5] J. S. Lee, "Refined filtering of image noise using local statistics," Comput. Graphics Image Process., vol. 15, no. 4, pp. 380-389, Apr. 1981.

[6] G. Vasile, E. Trouve, J.-S. Lee, and V. Buzuloiu, "Intensity-driven adaptive-neighborhood technique for polarimetric and interferometric SAR parameters estimation," IEEE Trans. Geosci. Remote Sens., vol. 44, no. 6, pp. 1609-1621, Jun. 2006.

[7] P. Salembier and L. Garrido, "Binary partition tree as an efficient representation for image processing, segmentation, and information retrieval," IEEE Trans. Image Process., vol. 9, no. 4, pp. 561-576, Apr. 2000.

[8] P. Heas and M. Datcu, "Modeling trajectory of dynamic clusters in image time-series for spatio-temporal reasoning," IEEE Trans. Geosci. Remote Sens., vol. 43, no. 7, pp. 1635-1647, Jul. 2005.

[9] L. Gueguen and M. Datcu, "Image time-series data mining based on the information-bottleneck principle," IEEE Trans. Geosci. Remote Sens., vol. 45, no. 4, pp. 827-838, Apr. 2007.

[10] S. Quegan, T. Le Toan, J. J. Yu, F. Ribbes, and N. Floury, "Multitemporal ERS SAR analysis applied to forest mapping," IEEE Trans. Geosci. Remote Sens., vol. 38, no. 2, pp. 741-753, Mar. 2000.

[11] L. Bruzzone, M. Marconcini, U. Wegmuller, and A. Wiesmann, "An advanced system for the automatic classification of multitemporal SAR images," IEEE Trans. Geosci. Remote Sens., vol. 42, no. 6, pp. 13211334, Jun. 2004.

[12] A. Julea, F. Ledo, N. Meger, E. Trouve, P. Bolon, C. Rigotti, R. Fallourd, J.-M. Nicolas, G. Vasile, M. Gay, O. Harant, L. Ferro-Famil, and F. Lodge, "Polsar Radarsat-2 Satellite Image Time Series mining over the Chamonix Mont-Blanc test site," in Proc. IEEE IGARSS, Jul. 2011, pp. 1191-1194.

[13] F. Harary, Graph Theory. Reading, MA, USA: Addison-Wesley, 1969.

[14] A. Alonso-Gonzalez, C. Lopez-Martinez, and P. Salembier, "Filtering and segmentation of polarimetric SAR images with binary partition trees," in Proc. IEEE IGARSS, Jul. 2010, pp. 4043-4046.

[15] A. Alonso-Gonzalez, C. Lopez-Martinez, and P. Salembier, "Filtering and segmentation of polarimetric SAR data based on binary partition trees," IEEE Trans. Geosci. Remote Sens., vol. 50, no. 2, pp. 593-605, Feb. 2012.

[16] A. Alonso-González, S. Valero, J. Chanussot, C. López-Martínez, and P. Salembier, "Processing multidimensional SAR and hyperspectral images with binary partition tree," Proc. IEEE, vol. 101, no. 3, pp. 723-747, Mar. 2013.

[17] A. Alonso-Gonzalez, C. Lopez-Martinez, and P. Salembier, "PolSAR speckle filtering and segmentation based on binary partition tree representation," in Proc. ESA PolInSAR, 2011, pp. 1-19.

[18] F. Barbaresco, "Interactions between symmetric cone and information geometries: Bruhat-tits and Siegel spaces models for high resolution autoregressive Doppler imagery," in Emerging Trends in Visual Computing, F. Nielsen, Ed. Berlin/Heidelberg, Germany: Springer-Verlag, 2009, ser. vol. 5416 of Lecture Notes in Computer Science, pp. 124-163.

[19] A. Alonso-Gonzalez, C. Lopez-Martinez, and P. Salembier, "Binary partition tree as a polarimetric SAR data representation in the space-time domain," in Proc. IEEE IGARSS, Jul. 2011, pp. 3819-3822.

[20] A. Alonso-Gonzalez and C. Lopez-Martinez, "Space-time PolSAR data temporal series processing based on binary partition trees," in Proc. 9th EUSAR, Apr. 2012, pp. 705-708.

[21] A. Alonso-Gonzalez, C. Lopez-Martinez, and P. Salembier, "Temporal polsar image series exploitation with binary partition trees," in Proc. IEEE IGARSS, Jul. 2012, pp. 1493-1496.

[22] S. R. Cloude and K. P. Papathanassiou, "Polarimetric SAR interferometry," IEEE Trans. Geosci. Remote Sens., vol. 36, no. 5, pp. 1551-1565, Sep. 1998.

[23] S. R. Cloude and E. Pottier, "A review of target decomposition theorems in radar polarimetry," IEEE Trans. Geosci. Remote Sens., vol. 34, no. 2, pp. 498-518, Mar. 1996.

[24] R. J. Muirhead, Aspects of Multivariate Statistical Theory. New York, NY, USA: Wiley, 1982. 
[25] C. Lopez-Martinez, E. Pottier, and S. R. Cloude, "Statistical assessment of eigenvector-based target decomposition theorems in radar polarimetry," IEEE Trans. Geosci. Remote Sens., vol. 43, no. 9, pp. 2058-2074, Sep. 2005.

[26] Google Earth. [Online]. Available: http://earth.google.com

[27] Panoramio. [Online]. Available: http://www.panoramio.com

[28] K. Conradsen, A. A. Nielsen, J. Schou, and H. Skriver, "A test statistic in the complex Wishart distribution and its application to change detection in polarimetric SAR data," IEEE Trans. Geosci. Remote Sens., vol. 41, no. 1, pp. 4-19, Jan. 2003.

[29] L. Pipia, X. Fabregas, A. Aguasca, and C. Lopez-Martinez, "Polarimetric temporal analysis of urban environments with a ground-based SAR," IEEE Trans. Geosci. Remote Sens., vol. 51, no. 4, pp. 2343-2360, Apr. 2013.

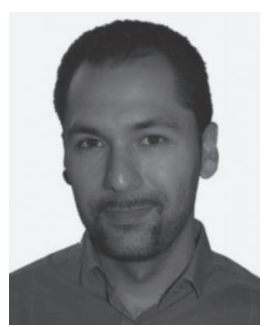

Alberto Alonso-González (S'11) was born in León, Spain, in 1984. He received the B.Sc. degree in computer science and the M.Sc. degree in telecommunication engineering from the Technical University of Catalonia (UPC), Barcelona, Spain, in 2007 and 2009 , respectively, where he is currently working toward the Ph.D. degree in telecommunication engineering.

In 2009, he joined the Signal Theory and Communications Department (TSC), Technical University of Catalonia. His Ph.D. thesis is focused on multidimensional synthetic aperture radar (SAR) data modeling and processing. His research interests include multidimensional SAR, SAR interferometry and polarimetry, digital signal and image processing, and data segmentation and simplification techniques.

Mr. Alonso-González was the recipient of the First Place Student Paper Award at the EUSAR 2012 Conference.

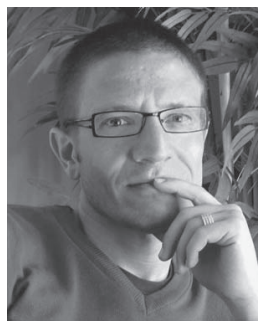

Carlos López-Martínez (S'97-M'04-SM'11) received the M.Sc. degree in electrical engineering and the Ph.D. degree from the Universitat Politècnica de Catalunya, Barcelona, Spain, in 1999 and 2003, respectively.

From October 2000 to March 2002, he was with the Frequency and Radar Systems Department (HR), German Aerospace Center (DLR), Oberpfaffenhofen, Germany. From June 2003 to December 2005, he has been with the Image and Remote Sensing GroupSAPHIR Team, in the Institute of Electronics and Telecommunications of Rennes (IETR - CNRS UMR 6164), Rennes, France. In January 2006, he joined the Universitat Politècnica de Catalunya as a Ramón-y-Cajal Researcher, where he is currently an Associate Professor in the area of remote sensing and microwave technology. His research interests include SAR and multidimensional SAR, radar polarimetry, physical parameter inversion, digital signal processing, estimation theory, and harmonic analysis.

Dr. López-Martínez is an Associate Editor of the IEEE JOURnal OF SELected Topics in Applied Earth Observations and Remote SensING, and he served as a Guest Editor of the EURASIP Journal on Advances in Signal Processing. He has organized different invited sessions in international conferences on radar and SAR polarimetry. He has presented advanced courses and seminars on radar polarimetry to a wide range of organizations and events. He was the recipient of the Student Prize Paper Award at the EUSAR 2002 Conference and coauthored the paper awarded with the First Place Student Paper Award at the EUSAR 2012 Conference.

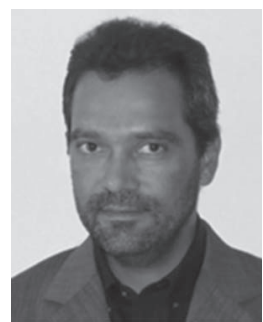

Philippe Salembier (M'96-SM'09-F'11) received the degree from the Ecole Polytechnique, Paris, France, in 1983 and a degree from the Ecole Nationale Supérieure des Télécommunications, Paris, in 1985. He received the Ph.D. degree from the Swiss Federal Institute of Technology (EPFL), Lausanne, Switzerland, in 1991.

He was a Postdoctoral Fellow at the Harvard Robotics Laboratory, Cambridge, MA, USA, in 1991. From 1985 to 1989, he worked at Laboratoires d'Electronique Philips, Limeil-Brevannes, France, in the fields of digital communications and signal processing for HDTV. In 1989 , he joined the Signal Processing Laboratory of the EPFL to work on image processing. At the end of 1991, after a stay at the Harvard Robotics Laboratory, he joined the Technical University of Catalonia, Barcelona, Spain, where he is currently a Professor lecturing on the area of digital signal and image processing. His current research interests include image and sequence coding, compression and indexing, segmentation, video sequence analysis, mathematical morphology, level sets, and nonlinear filtering. In terms of standardization activities, he has been particularly involved in the definition of the MPEG-7 standard ("Multimedia Content Description Interface") as the Chair of the "Multimedia Description Scheme" group between 1999 and 2001.

Prof. Salembier is a Fellow of the IEEE. He served as an Area Editor of the Journal of Visual Communication and Image Representation (Academic Press), Coeditor-in-Chief of Signal Processing (Eurasip), and Associate Editor of the IEEE Transactions on Image Processing and of the IEEE Signal PROCESSING LetTERs. Finally, he is currently an Associate Editor of the Eurasip Journal on Image and Video Processing, Elsevier Signal Processing. Image Communication, and the IEEE TRANSACTIONS ON CIRCUITS AND SYSTEMS FOR VIDEO TECHNOLOGY. He was a member of the Image and Multidimensional Signal Processing Technical Committee of the IEEE Signal Processing Society between 2000 and 2006. 